\title{
Raman investigation of air-stable silicene nanosheets on an inert graphite surface
}

Paola Castrucci ${ }^{1}(\bowtie)$, Filippo Fabbri ${ }^{2}(\bowtie)$, Tiziano Delise ${ }^{1}$, Manuela Scarselli ${ }^{1}$, Matteo Salvato ${ }^{1}$, Sara Pascale $^{3}$, Roberto Francini ${ }^{4}$, Isabelle Berbezier ${ }^{5}$, Christoph Lechner ${ }^{6}$, Fatme Jardali ${ }^{7}$, Holger Vach $(\bowtie)$, and Maurizio De Crescenzi ${ }^{1}$

1 Dipartimento di Fisica, Università di Roma "Tor Vergata", Roma 00133, Italy

${ }^{2}$ Center for Nanotechnology Innovation c/o NEST, Istituto Italiano di Tecnologia, Pisa 56127, Italy

${ }^{3}$ Consorzio di Ricerca Hypatia, c/o Italian Space Agency, Roma 00133, Italy

${ }^{4}$ Dipartimento di Ingegneria Industriale, Università di Roma "Tor Vergata", Roma 00133, Italy

${ }^{5}$ CNRS, Aix-Marseille Université, IM2NP, UMR 7334, Marseille 13397, France

${ }^{6}$ EDF R\&D, Department Materials and Mechanics of Components (MMC), Moret-sur-Loing, France

${ }^{7}$ CNRS-LPICM, Ecole Polytechnique, Université Paris-Saclay, Palaiseau 91128, France

\section{TABLE of CONTENT}
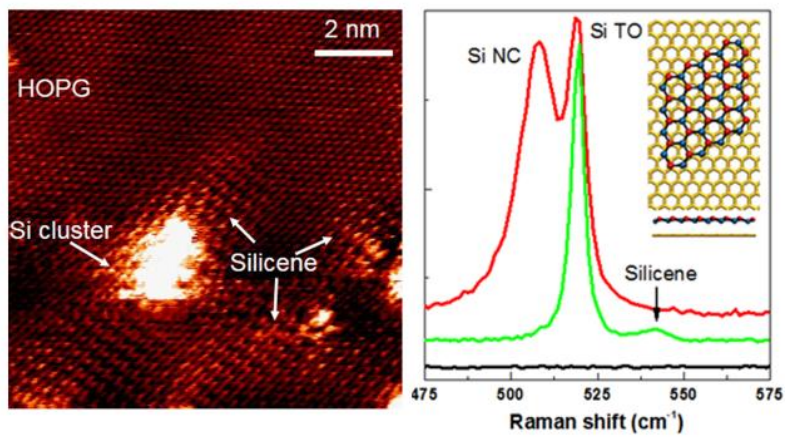

Identification of the Raman mode of air-stable low-buckled (with $\mathrm{sp}^{2}$ configuration) silicene nanosheets synthesized on highly oriented pyrolytic graphite located at $542.5 \mathrm{~cm}^{-1}$, perfectly reproduced by ab initio calculations. Scanning tunneling microscopy supports the growth of a low-buckled honeycomb structure 


\title{
Raman investigation of air-stable silicene nanosheets on an inert graphite surface
}

Paola Castrucci ${ }^{1}(\varangle)$, Filippo Fabbri ${ }^{2}(\varangle)$, Tiziano Delise ${ }^{1}$, Manuela Scarselli ${ }^{1}$, Matteo Salvato ${ }^{1}$, Sara Pascale $^{3}$, Roberto Francini ${ }^{4}$, Isabelle Berbezier ${ }^{5}$, Christoph Lechner ${ }^{6}$, Fatme Jardali ${ }^{7}$, Holger Vach ${ }^{7}$ $(\varangle)$, and Maurizio De Crescenzi ${ }^{1}$

1 Dipartimento di Fisica, Università di Roma "Tor Vergata", Roma 00133, Italy

${ }^{2}$ Center for Nanotechnology Innovation c/o NEST, Istituto Italiano di Tecnologia, Pisa 56127, Italy

${ }^{3}$ Consorzio di Ricerca Hypatia, c/o Italian Space Agency, Roma 00133, Italy

${ }^{4}$ Dipartimento di Ingegneria Industriale, Università di Roma "Tor Vergata", Roma 00133, Italy

${ }^{5}$ CNRS, Aix-Marseille Université, IM2NP, UMR 7334, Marseille 13397, France

${ }^{6}$ EDF R\&D, Department Materials and Mechanics of Components (MMC), Moret-sur-Loing, France

${ }^{7}$ CNRS-LPICM, Ecole Polytechnique, Université Paris-Saclay, Palaiseau 91128, France

\begin{abstract}
The fascinating properties of two dimensional (2D) crystals have gained increasing interest for many applications. The synthesis of a 2D silicon structure, namely silicene, is attracting great interest for possible development of next generation electronic devices. The main difficulty in working with silicene remains its strong tendency to oxidation when exposed to air as a consequence of its relatively highly buckled structure. In this work, we univocally identify the Raman mode of air-stable low-buckled silicene nanosheets synthesized on highly oriented pyrolytic graphite (HOPG) located at $542.5 \mathrm{~cm}^{-1}$. The main focus of this work is Raman spectroscopy and mapping analyses in combination with ab initio calculations. Scanning tunneling microscopy images reveal the presence of a patchwork of Si threedimensional (3D) clusters and contiguous $\mathrm{Si}$ areas presenting a honeycomb atomic arrangement, rotated by $30^{\circ}$ with respect to the HOPG substrate underneath, with a lattice parameter of $0.41 \pm 0.02 \mathrm{~nm}$ and a buckling of the Si atoms of $0.05 \mathrm{~nm}$. Raman analysis supports the co-existence of 3D silicon clusters and 2D silicene. The Raman shift of low-buckled silicene on an inert substrate has not been reported so far and it is completely different from the one calculated for free-standing silicene and the ones measured for silicene grown on $\mathrm{Ag}(111)$ surfaces. Our experimental results are perfectly reproduced by our $a b$ initio calculations of deposited silicene nanosheets. This leads us to conclude that the precise value of the observed Raman shift crucially depends on the strain between the silicene and the HOPG substrate.
\end{abstract}

\section{KEYWORDS}

2D Materials, Silicene, Raman spectroscopy, ab initio calculations, scanning tunneling microscopy, scanning tunneling spectroscopy.

Address correspondence to Paola Castrucci, email: castrucci@roma2.infn.it; Filippo Fabbri, email: filippo.fabbri@iit.it; Holger Vach, email: holger.vach@polytechnique.edu 


\section{Introduction}

Graphene, one of the allotropes of carbon, is an atomically flat single sheet which exhibits a honeycomb structure with $\mathrm{sp}^{2}$ hybridization of the carbon atoms [1]. Individual graphene layers were first isolated by cleaving graphite using "adhesive tape" [1, 2]. This material demonstrates superior electrical and mechanical properties, in fact displaying a room temperature quantum Hall effect $[3,4]$, and more importantly, it is the strongest material reported to date [4]. Graphene is a semimetal with its conduction band and valence band being degenerate at the K point in the Brillouin zone and has an extremely high room temperature carrier mobility (about 2 orders of magnitude greater than that of silicon) [5].

Although belonging to the same group as carbon, Si preferentially tends to arrange in $\mathrm{sp}^{3}$ configuration with its surrounding atoms. Even for an ultrathin film, silicon atoms deposited on a solid surface form three-dimensional (3D) diamond-like structures [6 - 8]. In spite of these generally accepted features, recent theoretical and experimental works have demonstrated the existence of a stable phase of singlelayered silicon with graphene-like structure (generally called silicene) in a slightly puckered configuration $[9,10]$. Silicene has been attracting much attention for the promising improvements that its use can imply in silicon based nano-electronic devices [11].

Raman spectroscopy of bulk silicon shows a main transverse optical (TO) peak located at $520 \mathrm{~cm}^{-1}$ while the free-standing (FS) silicene $E_{2 g}$ peak has been predicted to be between 549 and $575 \mathrm{~cm}^{-1}$ according to the calculation method used [12,13]. Recently, Raman spectra of silicene on Ag (111) substrates have revealed features markedly different from the previous ones which are located a) at around $514 \mathrm{~cm}^{-1}$ for $3 \times 3 / 4 \times 4$ epitaxial silicene on $\mathrm{Ag}(111)$ [14]; b) ranging between 515 and $522 \mathrm{~cm}^{-1}$ for $2 \sqrt{ } 3 \times 2 \sqrt{ } 3$ epitaxial silicene [11] and c) at around $530 \mathrm{~cm}^{-1}$ for epitaxial $\sqrt{ } 13 \times \sqrt{ } 13 / 4 \times 4$ silicene [15]. This wide range of results has been associated to differences in: surface reconstructions, the amount of silicene buckling and/or charge doping effects. Indeed, theoretical calculations support the observed red-shifting of the silicene $\mathrm{E}_{2 g}$ mode by increasing the buckling [16]. Recently, we have reported evidence that patches of silicene can grow on inert substrates such as highly oriented pyrolitic graphite (HOPG) [17]. The nanosheets of silicene have often been detected in the vicinity of small nanosized 3D Si clusters that are smaller than about $3 \mathrm{~nm}$ and that will be named Si nanoclusters hereafter leaving large parts of the HOPG surface free of additional nanostructures. This kind of surface inhomogeneity has also been reported for the growth of silicene nanosheets on $\mathrm{MoS}_{2}$ [18].

In this work, we univocally identify the Raman mode of air-stable low-buckled silicene nanosheets, grown on a HOPG substrate. We carefully studied the vibrational properties of the silicon/HOPG surface by acquiring Raman maps on the micrometric scale, demonstrating the coexistence of 3D silicon structures and low-buckled silicene nanosheets. We have observed specific features that can be assigned to the silicene $\mathrm{E}_{2 \mathrm{~g}}$ vibrational mode, Si nanoclusters and large silicon clusters, as well as the $\mathrm{E}_{2 \mathrm{~g}}$ mode of defect-free and unperturbed HOPG (namely, the G-band). We have found the first peak to be located around $542.5 \pm 0.5 \mathrm{~cm}^{-1}$. We interpret this silicene $\mathrm{E}_{2 \mathrm{~g}}$ value as being due to the low buckling (about 0.05 $\mathrm{nm}$ ) of silicon atoms in the honeycomb arrangement of silicene grown on a HOPG substrate, observed by scanning tunneling microscopy (STM). Until now, this value is markedly different from all the values reported in the literature for any other silicon configuration and for silicene on $\operatorname{Ag}(111)$ [11, 14, 15].

\section{Experimental}

A HOPG (from GE Advanced Ceramics, USA, $12 \mathrm{~mm} \times 12 \mathrm{~mm} \times 1 \mathrm{~mm}$ ) sample was used as a substrate. A fresh surface of graphite was obtained by peeling the HOPG substrate with adhesive tape and transferring it into a silicon (Sil'tronix ST, $\mathrm{Q}=1-10 \Omega \bullet \mathrm{cm}$, n-doped) was evaporated from a wafer located at $200 \mathrm{~mm}$ from the substrate. The deposition was achieved under UHV conditions (base pressure low 
$10^{-10}$ Torr $)$ and at a constant rate of $0.01 \mathrm{~nm} / \mathrm{min}(0.04 \mathrm{ML} / \mathrm{min})$ monitored by an Inficon quartz balance. Deposition was carried out keeping the substrate at room temperature (RT). STM imaging was performed using an Omicron-STM system with electrochemically etched tungsten tips. The STM was calibrated by acquiring atomically resolved images of the bare HOPG surface. All images were acquired in the constant current mode and were unfiltered apart from the rigid plane subtraction. Ex-situ Raman spectroscopy and mapping have been performed without capping the sample. The Raman analyses was carried out with a $532 \mathrm{~nm}$ excitation laser, a laser power density of $0.1 \mathrm{~mW} / \mu^{2}$ and an acquisition time of $1 \mathrm{~s}$. The spectral resolution is $2 \mathrm{~cm}^{-1}$. Low magnification line scans were performed with a 20X objective (NA 0.40), acquiring a spectrum every $200 \mu \mathrm{m}$. Meanwhile, the high magnification Raman maps were obtained with a 100X objective (NA=0.85), with $500 \mathrm{~nm}$ of lateral resolution. The Raman system is a Renishaw Invia Qotor equipped with a confocal optical microscope and a high resolution Andor CCD camera. Preliminary Raman characterization of the bare HOPG surface before Si deposition is reported in Fig. S1 of the Electronic Supplementary Material (ESM) file. From the Voigt deconvolution, it is possible to evaluate the peak position and FWHM and the associated error as 0.5 $\mathrm{cm}^{-1}$.

$\mathrm{Ab}$ initio calculations were performed with the CRYSTAL14 software package [19, 20]. The Perdew-Burke-Ernzerhof functional [21] combined with Grimme's D2 dispersion correction [22] (s6 = 0.25) was applied. Information regarding the basis set can be found in the ESM. For the infinite graphitesilicene interfaces, a four layer slab with AB stacking was used to model the HOPG surface. Each slab consisted of 14 and 128 carbon atoms for rotation angles of 10 and 30 degrees between the hexagonal patterns, respectively. The calculation of the binding energy is explained in the ESM. For finite nanoislands, the HOPG surface was reduced to one graphene layer consisting of 350 and 512 carbon atoms for 10 and 30 degrees, respectively. The silicene nano-islands consisted of 48 (zigzag) and 54 (armchair) $\mathrm{Si}$ atoms; the edge atoms were saturated with hydrogen. The energy and force thresholds for the optimizations and frequency calculations $[23,24]$ were $10^{-10}$ a.u. and $4.5 \times 10^{-5}$ a.u.. A $3 \times 3$ Monkhorst-Pack mesh including the $\Gamma$ point was used throughout except for FS silicene and the 10 degree interface where a 20x20 mesh was employed. The modes which stem from the $E_{2 g}$ mode of FS silicene were selected by calculating the maximum overlap (i.e. scalar product) between the displacement vectors of the $E_{2 g}$ mode of FS silicene and those of the interface/nano-islands. A thorough explanation of this approach can be found in the ESM.

\section{Results and Discussion}

\subsection{Sample characterization}

Figs. 1(a)-(c) show typical STM images, reported in a sequence with increasing magnification, of $1 \mathrm{ML}$ of Si deposited at RT on a HOPG surface. Si nanoclusters are homogeneously spread on darker and brighter areas of the HOPG surface, while they appear aligned decorating the straight steps of substrate terraces. In Fig. 1(d) the line profile, taken along the black line of the STM image in Fig. 1(c), displays the typical height of these Si nanoclusters that is about $0.5 \mathrm{~nm}$. In addition, it also exhibits the height of the brighter area on the HOPG surface with respect to the darker region, which amounts to $0.25 \pm 0.05$ nm. Similar values are obtained measuring line profiles (e.g. see Fig. 1(e)) in other zones of the surface. 

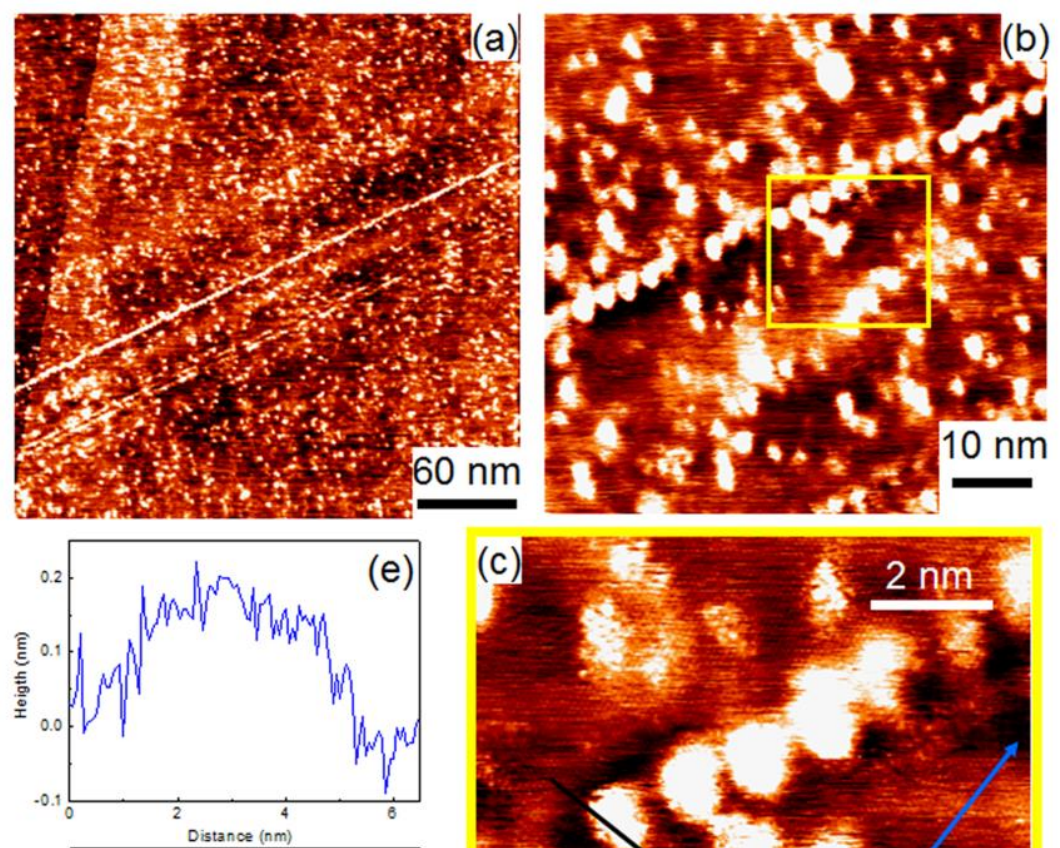

(e)

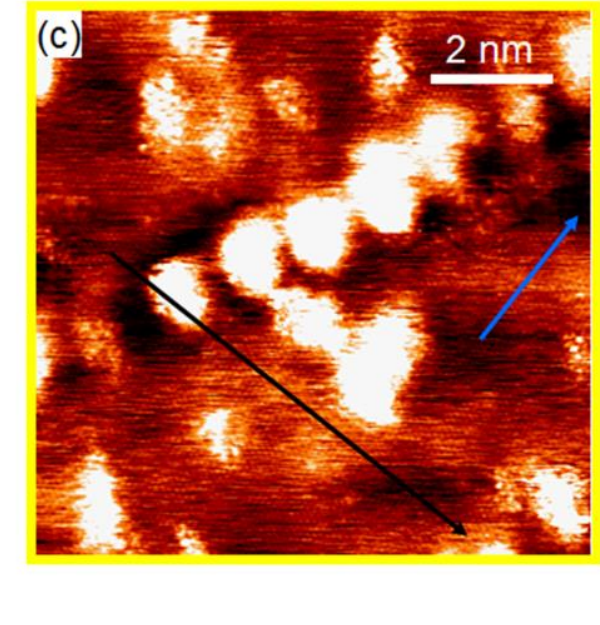

Figure 1 (a) Low magnification STM image $\left(\mathrm{V}_{\text {sample }}=+0.3 \mathrm{~V}, \mathrm{I}_{\mathrm{tunn}}=0.3 \mathrm{nA}\right)$ after one silicon monolayer deposited on a HOPG substrate at RT, showing the decoration of the atomic steps of HOPG by silicon nanoclusters. Larger magnification STM image (b) and (c) magnification of the yellow area highlighted in (b). (d) and (e) Height line profile along the black and blue arrows shown in (c), respectively. The areas which appear brighter with respect to the substrate are related to silicene nanosheets. The average height of flat silicon nanoclusters is less than $1 \mathrm{~nm}$.

Fig. 2 reports a blow-up around a few Si nanoclusters rising above flatter areas. These flatter regions appear like a patchwork of hexagonal patterns with different lattice spacing. Their extension, sometimes even larger than $6 \mathrm{~nm}$ (e.g. see Fig. 2a), prevents their interpretation in terms of quasi-particle interferences (QPI) or charge density waves induced on the HOPG surface by silicon clusters [25-27]. Moreover, line profiles measured along directions between the regions with different lattice parameters (see Fig. S2 in the ESM) show a steep height difference between these two areas of about 0.15-0.20 nm, which would not be expected in the QPI case [27]. In Fig. 2(b), we report a 3D STM image, displaying the different height of the surface areas and the Si nanoclusters. The bidimensional Fourier transform of the whole STM image reported in Fig. 2(a) shows two hexagonal spot patterns, one rotated by $30^{\circ}$ with respect to the other (see Fig. 2(c)). The distance $d$ between the spots and the center is $\sqrt{ } 3 / 2 a$, where $a$ is the hexagonal lattice parameter. The first Fourier pattern (outer hexagonal spots) gives rise to a lattice spacing typical of HOPG $(0.24 \pm 0.01 \mathrm{~nm})$. The inner hexagonal spots correspond to an $a$ value of $0.41 \pm 0.02 \mathrm{~nm}$, which is a factor $\sqrt{ } 3$ of the HOPG honeycomb lattice parameter. This is typical of a $(\sqrt{ }$ $3 \times \sqrt{ } 3) \mathrm{R}^{\circ} 0^{\circ} \mathrm{Si}$ superstructure arranged on the carbon atom graphitic monolayer as reported in Ref. [17] and [28]. In Fig. 2(d), we display a blow-up of the surface region with the largest lattice parameter. The honeycomb atomic arrangement on this surface is easily visible; a blue and red stick-and-ball model is superimposed on the STM experiment as guide to the eye. In Ref. [17], we have already reported that 


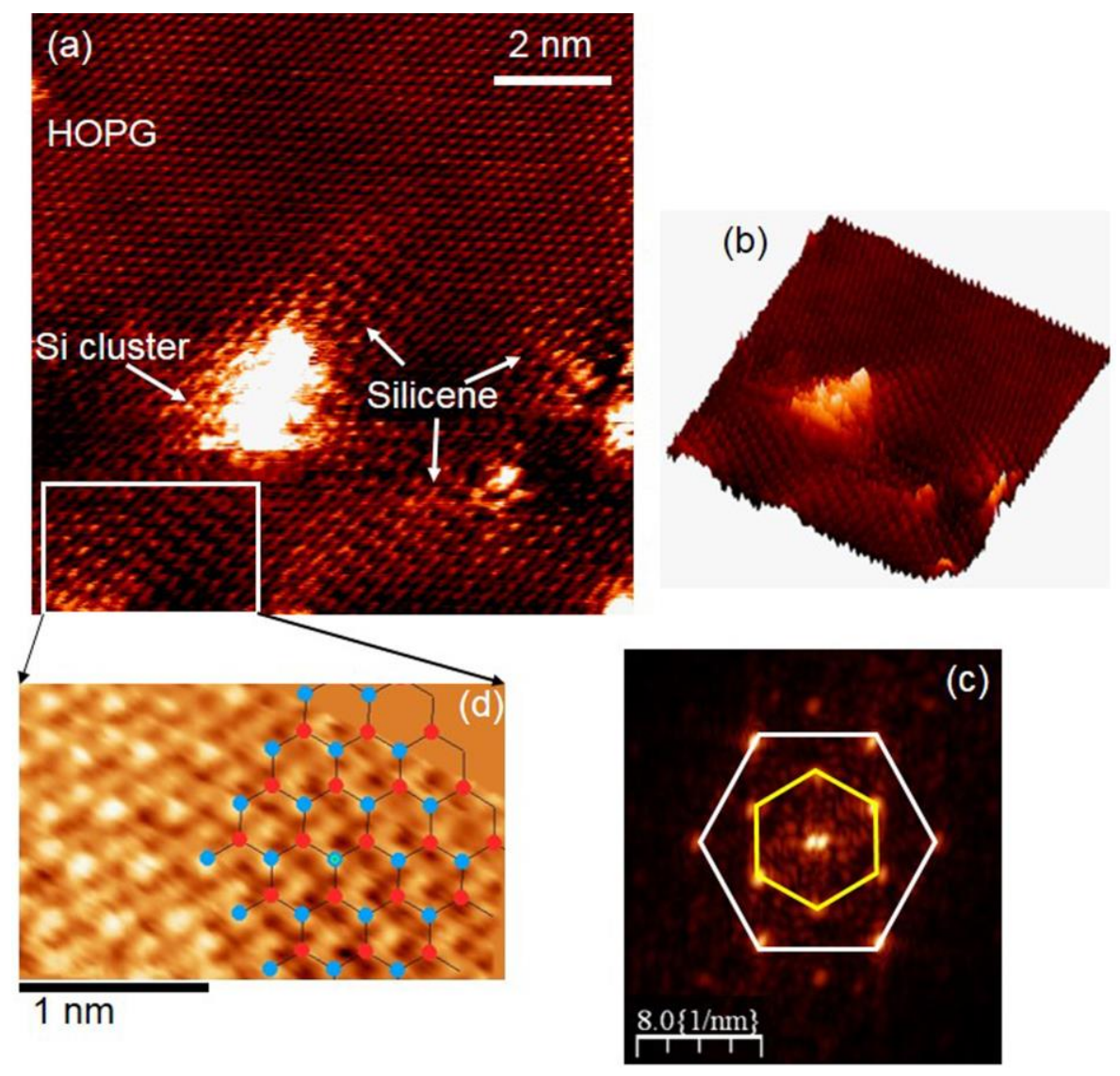

Figure 2 (a) STM images $\left(\mathrm{V}_{\text {sample }}=+0.3 \mathrm{~V}, \mathrm{I}_{\mathrm{tunn}}=0.3 \mathrm{nA}\right)$ after one silicon monolayer deposited on a HOPG substrate at RT. Note the coexistence of a silicene nanosheet and some silicon nanoclusters (white regions) with a height of less than $1 \mathrm{~nm}$. (b) Three dimensional reconstruction of image (a); (c) Bi-dimensional Fourier Transform of the STM image in panel (a): two hexagons (the external one due to HOPG and the internal one to silicene) rotated by about $30^{\circ}$ one with respect to the other. (d) Blow up of a particular area from (a) with the superimposition of the stick-and-ball model showing the honeycomb structure of silicene, blue and red atoms are slightly buckled by about $0.05 \mathrm{~nm}$ (the line profile displaying the height of the buckling has been already reported in ref. 17

the height difference of the atoms of the honeycomb lattice is $0.05 \pm 0.01 \mathrm{~nm}$. The presence of a buckling comparable with that of free-standing silicene [9] suggests that a low amount of interaction occurs between $\mathrm{Si}$ and $\mathrm{C}$ atoms. This is also confirmed by theoretical calculations, showing that there is a small amount of charge transfer from Si to $\mathrm{C}$ atoms (about $5 \times 10^{-4}$ electrons per atom) [28]. In excellent agreement to those arguments, we have calculated a binding energy between our silicene layer and the $\mathrm{HOPG}$ substrate that is about $40 \mathrm{meV} / \mathrm{Si}$ atom and that is mainly due to dispersion interactions. We like to point out, that this energy is strong enough for both to define a plane in which the silicene layer can grow and to hold it in place even above room temperature [17]. At the same time, however, this energy is weak enough to largely conserve the electronic properties of freestanding silicene. We also like to remind that this energy is quite comparable to the exfoliation energy of graphite of about 55.16 $\mathrm{meV} /$ atom and the exfoliation energy of $57.14 \mathrm{meV} /$ atom that was obtained when two graphene layers are exfoliated [29]. Our STM data support the results obtained by Persichetti et al. [30] and by L. Zhang et al. [31] indicating the formation of a few nanometer sized germanene nanosheets around 3D Ge clusters on HOPG or MoS 2 surface defects, respectively. It is worth noting that in both cases the authors detected a lattice parameter of the atomic network typical of germanene independently on the particular substrate. On the other hand, our STM results are completely different from the ones reported by Chiappe et al. [18] and by van Bremen et al. [32] where Si has been deposited on a MoS 2 inert substrate 
and STM images showed no lattice parameter enlargement occurring on silicene formation. While the former interpreted these data in terms of a squeezing of the silicene lattice producing a huge buckling, the latter suggested that a silicene layer intercalation occurred under the first atomic layer of MoS2.

\subsection{Raman analysis}

Considering the insights about the coexistence of 3D silicon structures contiguous to silicene nanosheets obtained by STM analysis, we performed low magnification Raman line-scan maps across the whole HOPG sample (Fig. S3 of ESM). We identify the three main Raman modes as the graphite G and D bands as well as the bulk silicon TO mode peaked at $1580 \mathrm{~cm}^{-1}, 1340 \mathrm{~cm}^{-1}$ and $520 \mathrm{~cm}^{-1}$, respectively. We use the bulk silicon TO mode as the benchmark for promising areas in which 3D silicon structures and silicene nanosheets coexist. Typical Raman spectra of bare HOPG and silicon decorated HOPG surfaces, extrapolated from Fig. S3 are reported in Fig. S4 of the ESM section.

Fig. 3(a) shows the typical Raman spectrum of three regions of the studied sample (green, red and black curves, for sake of clarity the spectra are shifted). The black curve corresponds to the Raman features of a bare HOPG substrate, with its typical G and D bands. The intensity ratio between D and G bands reveals an almost defect free HOPG, since the $G$ band dominates over the $D$ band which is generally ascribed to defects. [16] This also means that upon Si deposition, not all of the substrate surface is covered with silicon atoms. In the red and green curves, the Raman spectrum is conversely dominated by features located around $520 \mathrm{~cm}^{-1}$. The HOPG Raman features are still visible, but extremely reduced

a)

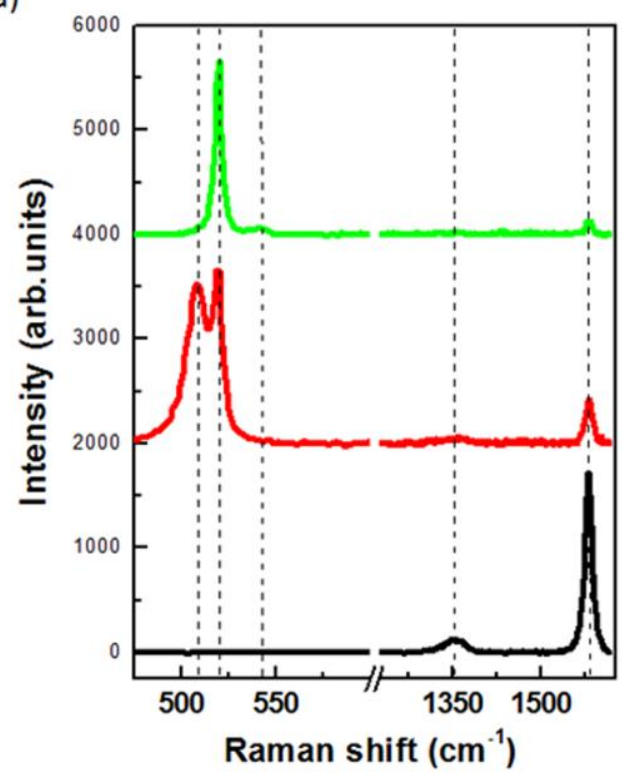

b)

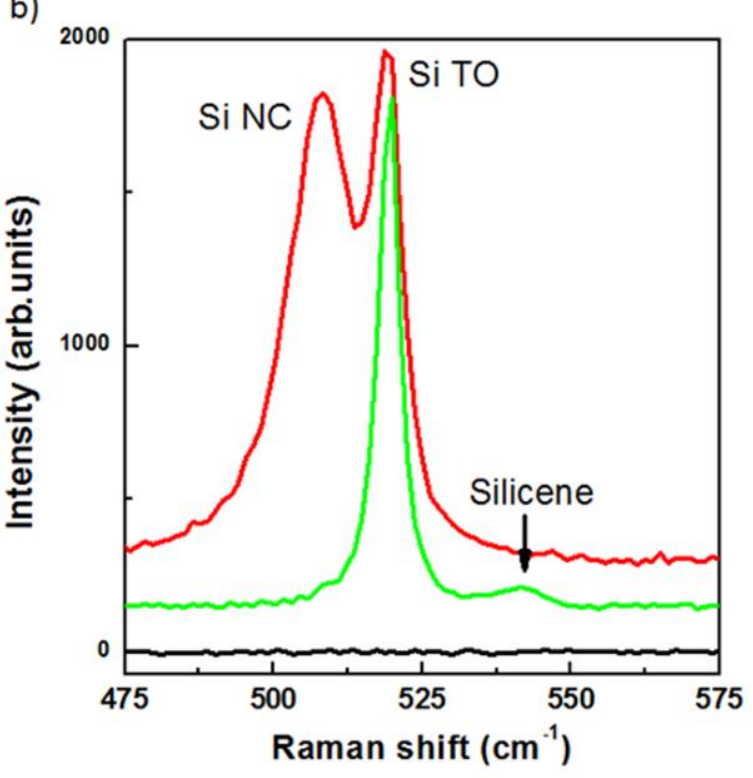

Figure 3 (a) Raman spectroscopy of promising areas of the HOPG sample after the deposition of 1 ML of silicon: the black line shows the standard Raman modes of bare graphite, the red line shows a peculiar Raman mode at $510 \mathrm{~cm}^{-1}$ due to quantum confined silicon nanoclusters (size less than $5 \mathrm{~nm}$ ) and at $520 \mathrm{~cm}^{-1}$ due to $\mathrm{Si} \mathrm{TO}$, and the green line shows the Raman resonance at $520 \mathrm{~cm}^{-1} \mathrm{due}$ to the TO Si mode; (b) A blow-up around $520 \mathrm{~cm}^{-1}$ where the green line clearly presents the Raman mode at $542.5 \mathrm{~cm}^{-1}$ which is due to the silicene nanosheets on top of the graphite substrate.

in intensity with respect to the dominant bulk Si resonance. Notably, no Raman feature located around $800 \mathrm{~cm}^{-1}$, where silicon carbide modes are expected [33], can be detected (see Fig. S4 in the ESM section). It is worth noting that, despite the absence of a capping layer, no sizeable Raman features due to silicon oxide are detectable in the spectra. [34]

Fig. 3(b) shows a blow-up around the Si $520 \mathrm{~cm}^{-1}$ resonance. Both the red and green curves exhibit no 
evidence of amorphous silicon, which typically appears as a broad band centered at $480 \mathrm{~cm}^{-1}$. [15] Both of these spectra can be fitted by using two Voigt curves, whose parameters are reported in Table S1 in ESM. The most intense Raman resonance peaks at $519.8 \mathrm{~cm}^{-1}$ and has a very narrow FWHM $\left(5 \mathrm{~cm}^{-1}\right)$, which is a typical for the bulk TO Si mode. [35] We ascribe this feature to large Si clusters. The peak located at $510 \mathrm{~cm}^{-1}$, with a FWHM as wide as $13.8 \mathrm{~cm}^{-1}$, could be assigned to Si nanoclusters with diameters smaller than the Bohr radius $(5 \mathrm{~nm})$, thus showing quantum confinement effects. [36] The attribution of these Raman modes to 3D Si structures (large Si clusters and Si nanoclusters) is corroborated by the presence of the weak, but wide feature extending between 900 and $1000 \mathrm{~cm}^{-1}$, which is reported to correspond to two-phonon scattering (2TO) coming from $\mathrm{Si}$ in the $\mathrm{sp}^{3}$ configuration (see Fig. S5 of ESM). [37] The feature located at $542.5 \mathrm{~cm}^{-1}$ has not been reported so far in the literature. Interestingly, its FWHM is as narrow as $7.2 \mathrm{~cm}^{-1}$ and it is fully Gaussian. This suggests that we are dealing with a genuine crystalline vibrational mode that we interpret as the silicene mode. In order to go deeper in the Raman analysis and try to establish the nature of this new mode, we report in Fig. 4 the Raman maps obtained by collecting hundreds of Raman spectra over a $26 \mu \mathrm{m} \times 21 \mu \mathrm{m}$ region. Each pixel size is $500 \mathrm{~nm} \times 500 \mathrm{~nm}$. In the top-left panel of Fig. 4, the optical image of the surface is reported while the other panels, from Fig. 4(b) to Fig 4(f), display the maps of the peaks of the G and D bands of HOPG located at $1580 \mathrm{~cm}^{-1}$ and $1340 \mathrm{~cm}^{-1}$ and of the intensity of the peaks centered at $520 \mathrm{~cm}^{-1}, 510 \mathrm{~cm}^{-}$ $1,542.5 \mathrm{~cm}^{-1}$ for the silicon structures, respectively.

Comparing these maps, we argue that the silicene Raman feature is quite diffused on the surface although it is not present everywhere (see Fig. 4(f)). However, it is prevalently found in surface areas close to large Si clusters and Si nanoclusters. Despite the giant difference in size between the areas

a) Optical Image

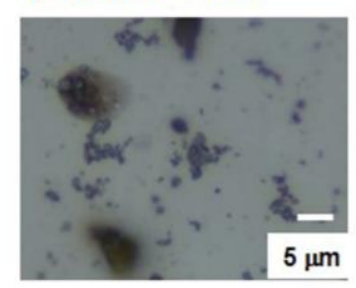

d) Si TO $520 \mathrm{~cm}^{-1}$

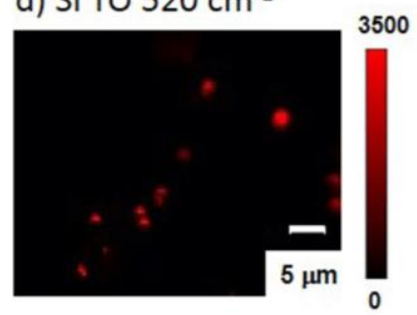

b) $\mathrm{G}$ band $1580 \mathrm{~cm}^{-1}$

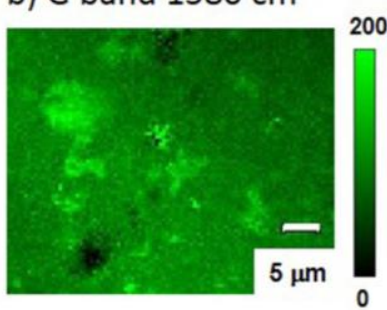

e) Si-NC $510 \mathrm{~cm}^{-1}$

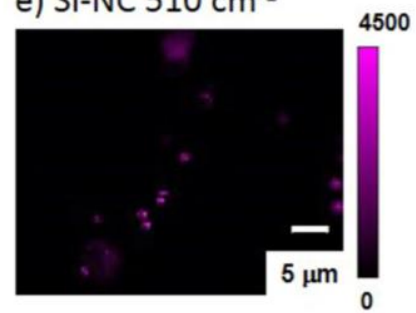

c) D band $1340 \mathrm{~cm}^{-1}$
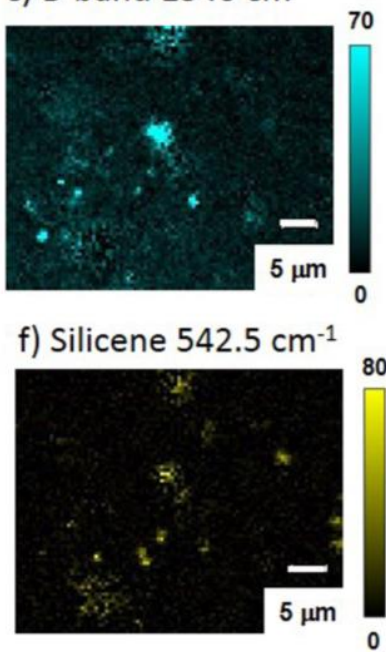

Figure 4 (a) High magnification confocal optical image (100X objective), (b) the G band intensity map, (c) the D Band intensity map, (d) the Si TO mode, (e) the mode of quantum confined silicon nanoclusters (Si-NC) and (f) the $\mathrm{E}_{2 \mathrm{~g}}$ mode of low-buckled silicene. Note that the silicene mode (yellow spots) is present in many areas of the surface, often but not always close to 3D Si structures.

probed by Raman spectroscopy and by STM, there is a surprising similarity between the information given by the two techniques. Moreover, thanks to this Raman mapping, we observe the silicene Raman resonances in sample areas where the amplitude of the D band Raman features slightly increases and no Si 3D structure Raman features are detected. We interpret this result as the possibility of silicene growth also occuring far away from large Si clusters and Si nanoclusters. From a theoretical point of view, stable free standing (FS) silicene has previously been reported to present the Raman $\mathrm{E}_{2 \mathrm{~g}}$ feature 
located at $575 \mathrm{~cm}^{-1}[12]$ and a buckling value of $0.044 \mathrm{~nm}$. However, there are calculations where by varying the buckling of the FS silicene layer (without addressing the energetic stability of the modified system), a downshift of the silicene $E_{2 g}$ feature is observed with increasing buckling [14]. If one refers to Fig. 1 of the paper of Scalise et al. [16], the Raman E2g peak for our STM experimental buckling of 0.05 $\mathrm{nm}$ is expected to occur around $542.8 \mathrm{~cm}^{-1}$. This Raman shift value fits very well with our experimental data (see Table S1 in ESM and Fig. 3(b)). Previous experimental Raman works showed an extreme reactivity of silicene grown on $\mathrm{Ag}(111)$ surfaces to oxygen, which compelled the investigators to measure the Raman spectra in-situ [14, 15] or to cap the sample for ex-situ measurements [11]. This observation occurs even in case of multilayer silicene samples [38]. Nevertheless, a Raman feature attributed to silicene remains clearly visible while other studies reported that after air exposure the Raman peak due to silicene disappears entirely after a short time [11]. We would like to underline the extreme air-stability of our sample after having exposed it to air for several weeks allowing us to perform the Raman measurements ex-situ without any capping. To understand this surprising airstability deeper, we made a series of checks of the possible formation of silicon oxide. First of all, we performed Raman maps for several wavenumbers at which $\mathrm{Si}-\mathrm{O}$ vibrational modes are expected, i.e. around $430 \mathrm{~cm}^{-1}, 600 \mathrm{~cm}^{-1}$ and $1065 \mathrm{~cm}^{-1}$ [34]. No relevant Raman amplitude can be detected for maps set at frequencies equal to $600 \mathrm{~cm}^{-1}$ and $1065 \mathrm{~cm}^{-1}$. Fig. 5a shows the Raman map obtained at $430 \mathrm{~cm}^{-1}$, which corresponds to the highest strength mode among the silicon oxide Raman features. The amplitude of this Raman mode is very low. In addition, one can observe a small signal in correspondence of areas of the sample mainly close to the silicene detected far from large Si clusters and Si nanoclusters. Furthermore, it looks as if the 3D Si structures are not affected by the oxidation process. To support these findings, we have performed X-ray photoemission spectroscopy measurements on the $2 \mathrm{p}$ Si core level before and after air-exposure of the HOPG sample covered with $1 \mathrm{ML}$ of silicon. Fig. 5b shows a comparison between the two XPS spectra. Interestingly, we observed no sizeable changes in the lineshape of $2 \mathrm{p} \mathrm{Si}$ after air-exposure. It is worth noting that the presence of silicon oxides is expected to produce a chemical shift up to $3.8 \mathrm{eV}$ [39]. This means that, within our XPS experimental resolution, no silicon oxide formation can be detected. Moreover, we recorded the STM images (Fig. 5c) of the sample surface after air-exposure and we are still able to observe the same morphology already shown in Fig. 2a, where a coexistence of Si nanoclusters and atomically resolved silicene (brighter region) and HOPG networks (darker areas) are visible. In Fig. 5d we exhibit a blow-up of the silicene region, indicated by the turquoise rectangular in Fig. 5c, showing the atomic resolution of these nanosheets after air exposure. Note the good coincidence with the stick and ball model of the silicene honeycomb lattice superimposed to the experimental STM image. We suggest that the very low reactivity with oxygen of silicene grown on HOPG compared to that grown on $\mathrm{Ag}(111)$ can be related to the difference in the buckling value. Indeed, in the latter case, the buckling has been estimated to vary between 0.07 and $0.1 \mathrm{~nm}$, a value considerably higher than the one measured in the present case $(0.05 \mathrm{~nm})$. This difference should give rise to a higher contribution of $\mathrm{sp}^{3}$ components in the silicon honeycomb structure in the latter case, thus favoring the presence of Si dangling bonds, which are highly reactive to oxygen. 

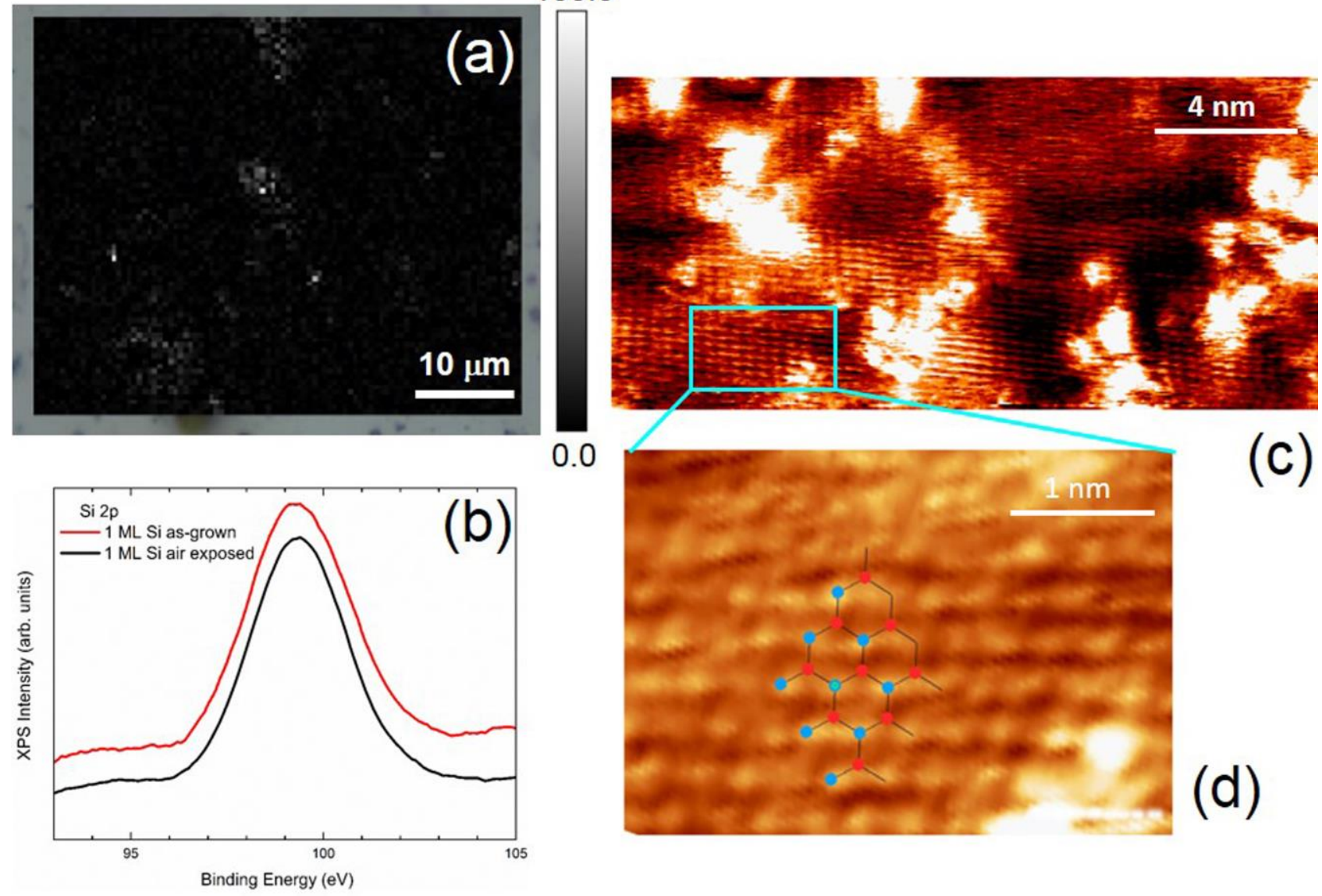

Figure 5 (a) High magnification Raman mode located at $430 \mathrm{~cm}^{-1}$, frequency generally ascribed to silicon oxides. This Raman map has been obtained in the same surface region already reported in Fig. 4. Compare the position of silicon oxides with the modes due to SiNCs, silicene and carbon D and G band (b) XPS Si 2p level spectra before and after air-exposure. No differences between the two spectra are detected within the experimental resolution. In particular, no feature due to silicon oxide and suboxides is observed. The two curves have been arbitrarily normalized and shifted for clarity. (c) STM image of the sample after air-exposure. Note the presence of Si nanoclusters (brightest spots whose height is very similar to that reported in Fig. 1), silicene nanosheets (brighter regions) and graphite substrate (darker areas). The difference in height between these two regions is less than $1 \mathrm{~nm}$, as obtained before exposure to air. (d) Blow-up of the brighter region indicated by the turquoise rectangular, showing the atomic resolution in the silicene nanosheet after air exposure. Note the good coincidence with the stick and ball model of the silicene honeycomb lattice superimposed to the experimental STM image.

Our air stability results are even better than those recently reported in Ref. [40] on quasi-freestanding epitaxial silicene grown on $\operatorname{Ag}(111)$ obtained by oxygen intercalation. The latter study has shown a gradual decrease in intensity of the Raman $\mathrm{E}_{2 \mathrm{~g}}$ feature with time and its complete disappearance after 6 days of air exposure. Concerning the exceptional stability of the Si 3D structures upon air-exposure, it is known that small Si nanoclusters are much less reactive to oxygen than the bulk because of the high energy activation barrier that increases up to a factor of four with the decrease in Si 3D structure size [41]. Moreover, in Fig. 6 we report the scanning tunneling spectroscopy (STS) for these small Si nanoclusters before and after air-exposure.

The V/I dI/dV curves shown in Fig. 6 are proportional to the filled and empty density of states close to 


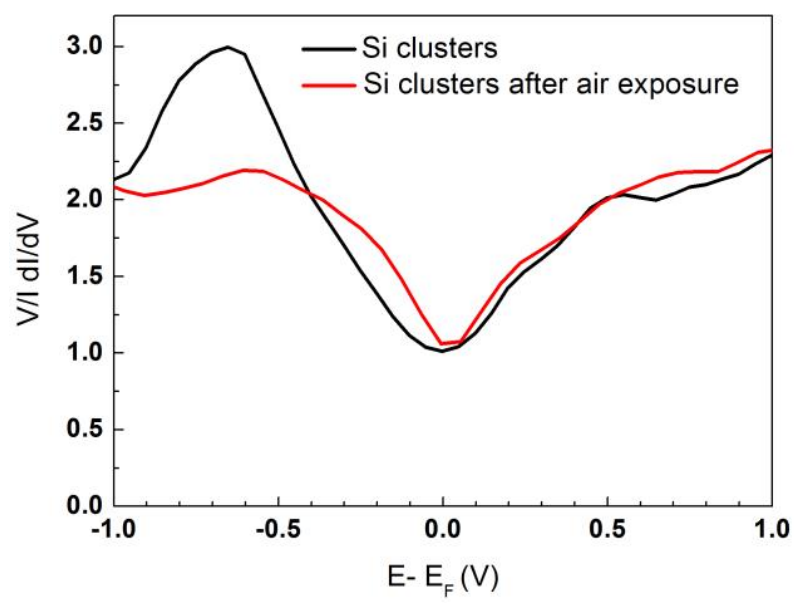

Figure 6 (a) V/I dI/dV of the current-voltage (I-V) curves recorded on Si nanoclusters before (black curve) (see Fig. 1b and 1c) and after (see Fig. 5c) air-exposure (red curve). The V/I dI/dV curves are proportional to the electronic density of empty and filled states. Note the metallic character of both the curves, due to the finite value of the density of states close to the Fermi level $\left(\mathrm{E}_{\mathrm{F}}\right)$.

the Fermi level (Е户) [17]. Due to non-zero value assumed by both the curves close to the Fermi level, we conclude that these Si nanoclusters are metallic in nature and remain metallic after air-exposure. The metal nature of very small Si nanoclusters is theoretically expected when no H-saturation of the surface dangling bonds is present [7]. To support our findings of silicene on HOPG, we have calculated theoretical spectra of these structures. To validate the applied ab initio method, we have first calculated the spectrum of FS silicene; the $E_{2 g}$ peak is found at $556 \mathrm{~cm}^{-1}$ and lies in the range of published values from Refs. [12, 13]. The Si-Si bond length is $0.229 \mathrm{~nm}$ and theand the buckling is $0.049 \mathrm{~nm}$. Two interfaces of silicene and HOPG were studied, the rotation angles of the two hexagonal patterns were 10 and 30 degrees, respectively. The modes which correspond to the $\mathrm{E}_{2 \mathrm{~g}}$ mode are shown in Table 1. For both interfaces a shortening of Si-Si bonds $(0.226 \mathrm{~nm}$ and $0.227 \mathrm{~nm}$ for 10 and 30 degrees, respectively) is observed in order to create a superlattice with HOPG. This also causes a slightly larger buckling of 0.060 and $0.057 \mathrm{~nm}$ for 10 and 30 degrees, respectively. For 10 degrees, a blueshift of the $E_{2 \mathrm{~g}}$ modes to $582 \mathrm{~cm}^{-}$ ${ }^{1}$ is found; however for 30 degrees, we observe a redshift to $540-544 \mathrm{~cm}^{-1}$. Experimentally, the pattern with a rotational angle of 30 degrees is dominant, thus we find an excellent agreement between theory and experiment. One reason for the difference between the interfaces are the structural parameters. Shortening of bond lengths leads to a blueshift, while increased buckling leads to a redshift, where the former is more pronounced. For FS silicene, a change of bond length from 0.227 to $0.226 \mathrm{~nm}$ (with constant buckling) blueshifts the frequency by $10 \mathrm{~cm}^{-1}$, while changing the buckling from 0.057 to 0.060

\begin{tabular}{|c|c|c|}
\hline & Mode 1 $\left(\mathrm{cm}^{-1}\right)$ & Mode 2 $\left(\mathrm{cm}^{-1}\right)$ \\
\hline Silicene & \multicolumn{2}{|c|}{555.6} \\
\hline IF-10 & 584.1 & 582.9 \\
\hline IF-30 & 539.8 & 546.3 \\
\hline NI-AC-10 & 542.4 & 550.8 \\
\hline NI-AC-30 & 550.5 & 542.1 \\
\hline NI-ZZ-10 & 546.5 & 546.2 \\
\hline NI-ZZ-30 & 545.9 & 545.5 \\
\hline
\end{tabular}

Table 1 Theoretical Raman shifts for several silicene configurations: Theoretical frequencies in $\mathrm{cm}^{-1}$ for the two Raman-active modes for free-standing silicene (Silicene), the infinite interfaces at 10 (IF-10) and 30 (IF-30) degrees rotational angle, the armchair edge nano-islands with 10 (NI-AC-10) and 30 (NI-AC-30) degrees rotational angle and zigzag nano-islands with 10 (NI-ZZ-10) and 30 (NI-ZZ-30) degrees rotational angle. 
$\mathrm{nm}$ redshifts it only by $1 \mathrm{~cm}^{-1}$. The difference of shifts for 10 and 30 degrees indicates that the Raman frequencies are very sensitive to strain in silicene caused by pattern creation with the HOPG substrate. However, the experimentally observed silicene nanosheets have a finite size whereas all previous calculations consider infinite systems only. In order to evaluate the influence of strain for finite size silicene, we calculated the spectra of four nano-islands of silicene (see Fig. 7) with side lengths of 1.3-1.5 $\mathrm{nm}$ and similar shape as in Fig 2(d), which differ in the edge type (zigzag and armchair) and rotation angle (10 and 30 degrees). The average bond length increases to $0.228 \mathrm{~nm}$ (for all nano-islands) due to the removal of periodic constraints with respect to the interfaces; buckling is reduced to $0.052-0.053 \mathrm{~nm}$ after optimization. We obtain a redshift for all nano-islands to about $542-550 \mathrm{~cm}^{-1}$ (see Fig. S7 in ESM for pictures of the modes). These theoretical results agree very well with both our experiments and our values calculated for the infinite silicene layer at 30 degrees. We like, therefore, to conclude that the IF30 is likely to experience a similar amount of strain as the nano islands which resemble the real experimentally observed system more closely.

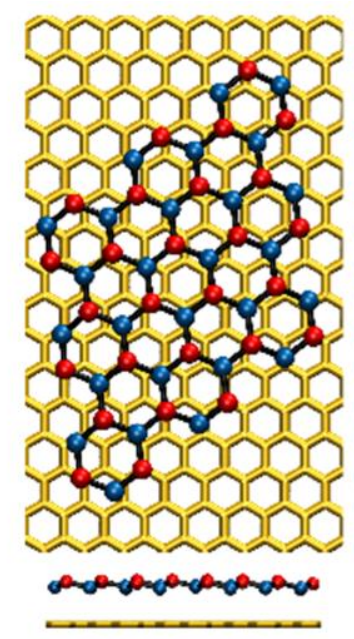

(a)

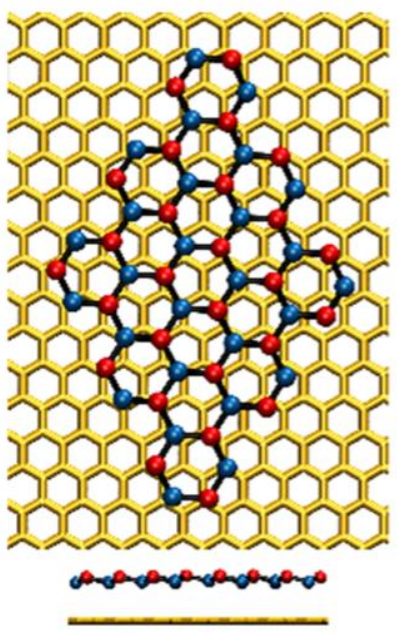

(b)

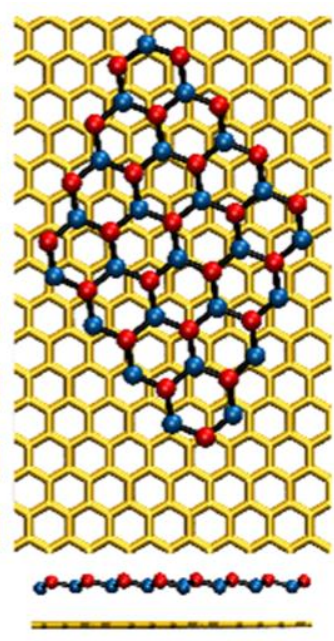

(c)

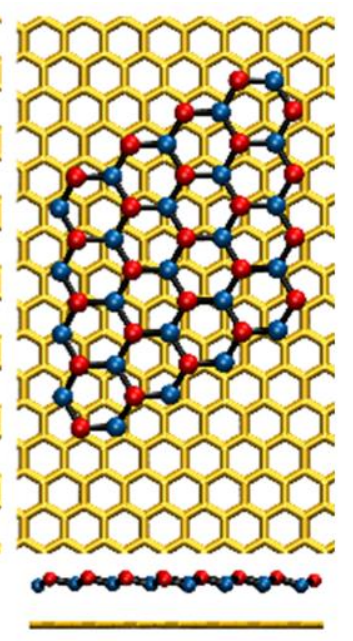

(d)

Figure 7 Optimized structures of armchair edge nano-islands with (a) 10 degrees and (b) 30 degrees rotational angle and zigzag nano-islands with (c) 10 degrees and (d) 30 degrees rotational angle; hydrogen atoms are omitted for better visibility, higher and lower $\mathrm{Si}$ atoms due to buckling are shown in red and blue, respectively.

\section{Conclusions}

In conclusion, though the growth mechanism of $\mathrm{Si}$ on the HOPG surface is prevalently threedimensional, our present study confirms that silicene nanosheets can be observed upon silicon deposition at room temperature on such an inert substrate. Raman spectra show, in fact, the appearance of a new feature located at $542.5 \mathrm{~cm}^{-1}$ never reported so far in the literature. The present Raman peak of silicene is located markedly far in wavenumbers from those reported for the several phases of silicene grown on $\mathrm{Ag}(111)$ surfaces. Moreover, this Raman resonance value is different from all previously observed ones for all known Si configurations, including amorphous silicon, 3D Si structures of any size, and bulk Si in $\mathrm{sp}^{3}$ configurations. Based on our ab initio calculations, we attribute the measured Raman shift to the strain that the finite size silicene nanosheets experience due to the interaction with the graphite substrate. Most importantly, we have shown for our small nano sheets that silicene grown on an inert substrate like HOPG is remarkably stable in air for at least several weeks, which we attribute to the observed low buckling and the resulting small number of dangling bonds. 


\section{Acknowledgements}

H.V., F.J., and C.L. gratefully acknowledge the HPC centers of the EDF, IDRIS (Grant i2016-090642), and CERMM for computational resources and the Hariri Foundation for Sustainable Human Development for the scholarship awarded to F.J.. M.D.C., P.C., M.S. and M.S. would like to acknowledge the European Community for the RISE Project CoExAN GA644076 and the project "Silicene and Germanene: novel two-dimensional nanomaterial" funded by the University of Roma Tor Vergata (Italy).

The authors acknowledge the original source of publication at https://doi.org/10.1007/s12274-018-2097$\underline{6}$.The original publication is available at www.springerlink.com

Electronic Supplementary Material: Supplementary material (please give brief details, e.g., further details of the annealing and oxidation procedures, STM measurements, AFM imaging and Raman spectroscopy measurements) is reported in the following.

\section{References}

[1] Novoselov, K. S.; Geim, A. K.; Morozov, S. V. ; Jiang, D.; Zhang, Y.; Dubonos, S. V.; Grigorieva, I. V.; Firsov, A. A. Electric field effect in atomically thin carbon films. Science 2004, 306, 666-669.

[2] Novoselov, K. S.; Geim, A. K.; Morozov, S. V.; Jiang, D. ; Katsnelson, M. I. ; Grigorieva, I. V. ; Dubonos, S. V. ; Firsov, A. A. Two-Dimensional Gas of Massless Dirac Fermions in Graphene. Nature 2005, 438, 197200.

[3] Novoselov, K. S.; Jiang, Z. ; Zhang, Y. ; Morozov, S. V.; Stormer, H. L.; Zeitler, U.; Maan, J. C.; Boebinger, G. S.; Kim, P.; Geim, A. K. Room-temperature quantum Hall effect in graphene. Science 2007, 315, 13791379.

[4] Lee, C.; Wei, X.; Kysar, J. W.; Hone, J. Measurement of the Elastic Properties and Intrinsic Strength. Science 2008, 321, 385-388.

[5] Geim, A. K.; Novoselov, K. S. The Rise of Graphene. Nat. Mater. 2007, 6, 183-191.

[6] Scheier, P.; Marsen, B.; Lonfat, M.; Schneider, W.-D.; Sattler, K. Growth of silicon nanostructures on graphite. Surf. Sci. 2000, 458, 113-122.

[7] Mélinon, P;. Kéghélian, P.; Prével, B.; Perez, A.; Guiraud, G.; LeBrusq, J.; Lermé, J.; Pellarin, M.; Broyer, M. Nanostructured silicon films obtained by neutral cluster depositions. J. Chem. Phys. 1997, 107, 10278-10287.

[8] Katircioglŭ, Ș.; Erkoç, Ș. Structural and electronic properties of bare and hydrogenated silicon clusters. Physica E 2001, 9, 314-320.

[9] Cahangirov, S.; Topsakal, M.; Akturk, E.; Sahin, H.; Ciraci, S. Two- and one-dimensional honeycomb structures of silicon and germanium. Phys. Rev. Lett. 2009, 102, 236804.

[10] Vogt, P.; De Padova, P.; Quaresima, C.; Avila, J.; Frantzeskakis, E.; Asensio, M. C.; Resta, A.; Ealet, B.; Le Lay, G. Silicene: Compelling Experimental Evidence for Graphenelike Two-Dimensional Silicon. Phys. Rev. Lett. 2012, 108, 155501.

[11]Tao, L.; Cinquanta, E.; Chiappe, D.; Grazianetti, C.; Fanciulli, M.; Dubey, M.; Molle, A.; Akinwande, D. Silicene field-effect transistors operating at room temperature. Nat. Nanotechnol. 2015, 10, 227-231.

[12] Scalise, E.; Houssa, M.; Pourtois, G.; van den Broek, B.; Afanas'ev, V.; Stesmans A. Vibrational properties of silicene and germanene. Nano Research 2013, 6, 19-28.

[13] Yan, J.-A.; Stein. R.; Schaefer, D. M.; Wang, X.-Q.; Chou, M. Y. Electron-phonon coupling in twodimensional silicene and germanene. Phys. Rev. B 2013, 88, 121403(R).

[14]Zhuang, J.; Xu, X.; Du, Y.; Wu, K.; Chen, L.; Hao, W.; Wang, J.; Yeoh, W. K.; Wang, X.; Dou, S. X. Investigation of electron-phonon coupling in epitaxial silicene by in situ Raman spectroscopy. Phys. Rev. B 2015, 91, 161409(R). 
[15] Solonenko, D.; Gordan, O. D.; Le Lay, G. ; Sahin, H. ; Cahangirov, S. ; Zahn, D. R. T. ; Vogt, P. 2D vibrational properties of epitaxial silicene on $\mathrm{Ag}(111) .2 \mathrm{D}$ Mater. 2016, 4, 015008.

[16] Scalise, E. ; Cinquanta, E. ; Houssa, M. ; van den Broek, B. ; Chiappe, D. ; Grazianetti, C. ; Pourtois, G. ; Ealet, B. ; Molle, A. Fanciulli M, Afanas'ev V V , Stesmans A. Vibrational properties of epitaxial silicene layers on (111) Ag. Appl. Surf. Sci. 2014, 291, 113- 117.

[17] De Crescenzi, M.; Berbezier, I.; Scarselli, M.; Castrucci, P. ; Abbarchi, M. ; Ronda, A. ; Jardali, F.; Park, J. ; Vach, H. Formation of Silicene Nanosheets on Graphite. ACS Nano 2016, 10, 11163-11171.

[18] Chiappe, D.; Scalise, E.; Cinquanta, E.; Grazianetti, C.; van den Broek, B.; Fanciulli, M.; Houssa, M.; Molle, A. Two-Dimensional Si Nanosheets with Local Hexagonal Structure on a MoS 2 Surface. Adv. Materials 2014, 26, 2096-2101.

[19] Dovesi, R. ; Civalleri, B. ; Roetti, C. ; Saunders, V. R. ; Zicovich-Wilson, C. M. CRYSTAL: A computational tool for ab initio study of the electronic properties of crystals. Z. Kristallogr. 2005, 220, 571-573.

[20] Dovesi, R.; Saunders, V. R.; Roetti, C.; Orlando, R.; Zicovich-Wilson, C. M.; Pascale, F.; , Civalleri, B.; Doll, K.; Harrison, N. M.; Bush, J. J.; D' Arco, Ph.; Llunell, M. CRYSTAL09 User's Manual (University of Torino, Torino, 2009).

[21] Perdew, J. P.; Burke, K.; Ernzerhof, M. Generalized Gradient Approximation Made Simple. Phys. Rev. Lett. 1996, 77, 3865-3868.

[22] Grimme, S. Semiempirical GGA-Type Density Functional Constructed with a Long-Range Dispersion Correction. J. Comput. Chem. 2006, 27, 1787-1799.

[23] Pascale, F.; Zicovich-Wilson, C. M.; Lopez, F.; Civalleri, B.; Orlando, R.; Dovesi R. The Calculation of the Vibration Frequencies of Crystalline Compounds and Its Implementation in the CRYSTAL Code. J. Comput. Chem. 2004, 25, 888-897.

[24]Zicovich-Wilson, C. M.; Pascale, F.; Roetti, C.; Saunders, V. R.; Orlando, R.; Dovesi, R. The Calculation of the Vibration Frequencies of alpha-Quartz: The Effect of Hamiltonian and Basis Set. J. Comput. Chem. 2004, 25, 1873-1881.

[25]Ferro, Y.; Thomas, C.; Angot, T.; Génésio, T.; Allouche, A. Theoretical and experimental characterization of damaged graphite surfaces. Journal of Nuclear Materials 2007, 363-365, 1206-1210.

[26] Büttner, M.; Choudhury, P.; Johnson, J. K.; Yates, J. T. Jr. Vacancy clusters as entry ports for cesium intercalation in graphite. Carbon 2011, 49, 3937-3952.

[27] Peng, W.; Xu, T.; Diener, P.; Biadala, L.; Berthe, M.; Pi, X.; Borensztein, Y.; Curcella, A.; Bernard, R.; Prévot, G.; Grandidier, B. Resolving the Controversial Existence of Silicene and Germanene Nanosheets Grown on Graphite. ACS Nano 2018, DOI: 10.1021/acsnano.8b01467

[28] Cai, Y.; Chuu, C.-P.; Wei, C. M.; Chou, M. Y. Stability and electronic properties of two-dimensional silicene and germanene on graphene. Phys. Rev. B 2013, 88, 245408.

[29] Chen, X.; Tian, F.; Persson, C.; Duan, W.; \& Chen, N. X. Interlayer interactions in graphites Sci. Rep. 2013, 3, 3046.

[30] Persichetti, L.; Jardali, F.; Vach, H.; Sgarlata, A.; Berbezier, I.; De Crescenzi, M.; \& Balzarotti, A. van der Waals Heteroepitaxy of Germanene Islands on Graphite. J. Phys. Chem. Lett. 2016, 7, 3246-3251.

[31]Zhang, L.; Bampoulis, P.; Rudenko, A. N.; Yao, Q.; van Houselt, A.; Poelsema, B.; Katsnelson, M. I. \& Zandvliet, H. J. W. Structural and Electronic Properties of Germanene on MoS2. Phys. Rev. Lett. 2016, 116, 256804.

[32] van Bremen, R.; Yao, Q.; Banerjee, S.; Cakir, D.; Oncel, N.; \& Zandvliet, H. J. W. Intercalation of Si between MoS2 layers. Beilstein J. Nanotechnol. 2017, 8, 1952-1960.

[33] Nakashima, S.; Harima, H. Raman Investigation of SiC Polytypes. Phys. Stat. Sol. (a) 1997, 162, 39-64.

[34] Borowicz, P.; Latek, M.; Rzodkiewicz, W.; Łaszcz, A.; Czerwinski, A.; Ratajczak, J. Deep-ultraviolet Raman investigation of silicon oxide. Adv. Nat. Sci.: Nanosci. Nanotechnol. 2012, 2, 045003.

[35] Parker, J. H.; Feldman, D. W.; Ashkin, M. Raman Scattering by Silicon and Germanium. Physical Review 1967, 155, 712-714.

[36] Faraci, G.; Gibilisco, S.; Pennisi, A. R. Quantum confinement and thermal effects on the Raman spectra of Si nanocrystals. Phys. Rev. B 2009, 80, 193410. 
[37] Temple, P. A.; Hathaway, C. E. Multiphonon Raman Spectrum of Silicon. Phys. Rev. B 1973, 7, 3685.

[38] De Padova, P.; Ottaviani, C.; Quaresima, C.; Olivieri, B.; Imperatori, P.; Salomon, E.; Angot, T.; Quaglian, L.; Romano, C.; Vona, A.; Muniz-Miranda, M.; Generosi, A.; Paci, B.; Le Lay, G. 24 h stability of thick multilayer silicene in air. 2D Materials 2014, 1, 021003.

[39] Himpsel, F. J.; McFeely, F. R.; Taleb-Ibrahimi, A.; Yarmoff, J. A.; Hollinger, G. Microscopic structure of the $\mathrm{SiO}_{2} / \mathrm{Si}$ interface. Phys. Rev. B 1988, 38, 6084.

[40] Du, Y.; Zhuang, J.; Wang, J.; Li, Z.; Liu, H.; Zhao, J.; Xu, X.; Feng, H.; Chen, L.; Wu, K.; Wang, X.; Dou, S. X. Quasi-freestanding epitaxial silicene on Ag (111) by oxygen intercalation. Sci. Adv. 2016, 2, e1600067.

[41]Jarrold, M. F.; Ray, U.; Creegan, K. M. Chemistry of semiconductor clusters: Large silicon clusters are much less reactive towards oxygen than the bulk. J. Chem. Phys. 1990, 93, 224. 


\section{Electronic Supplementary Material}

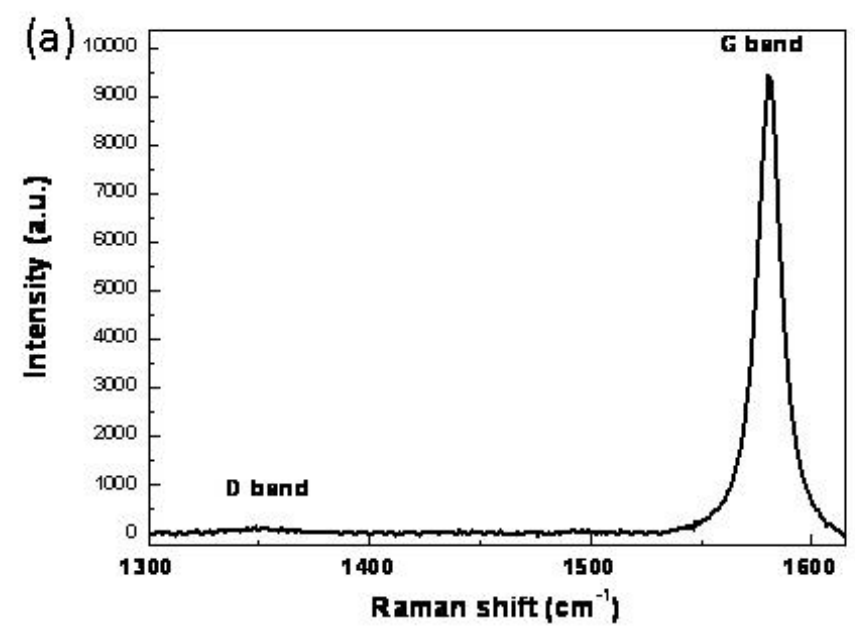

(b)

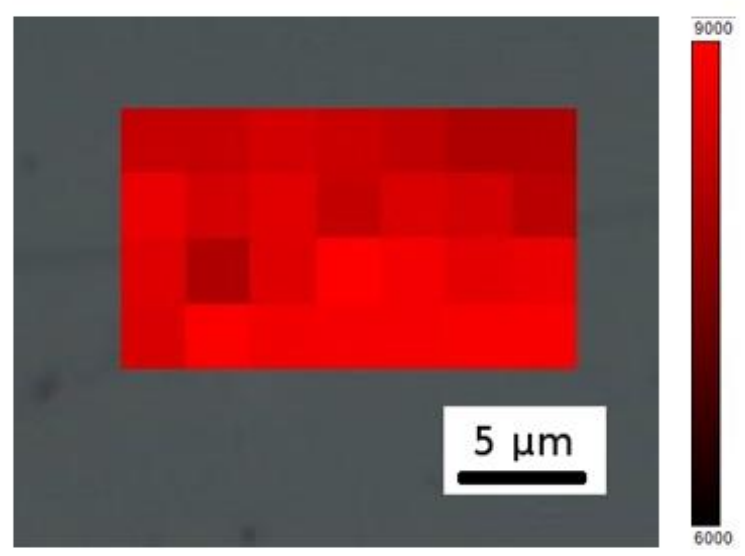

(c)

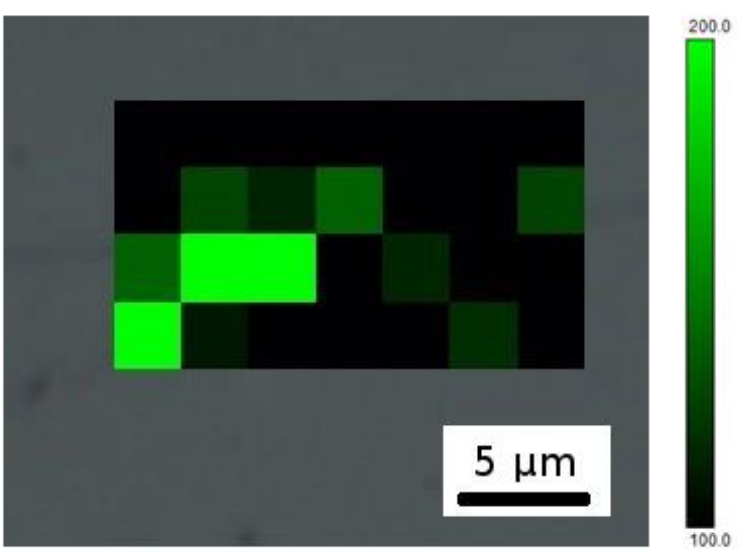

Figure S1 (a) Raman spectrum of pristine HOPG before silicon deposition, (b) G band (1580 $\left.\mathrm{cm}^{-1}\right)$ map, (c) D band $\left(1340 \mathrm{~cm}^{-1}\right)$ map. 

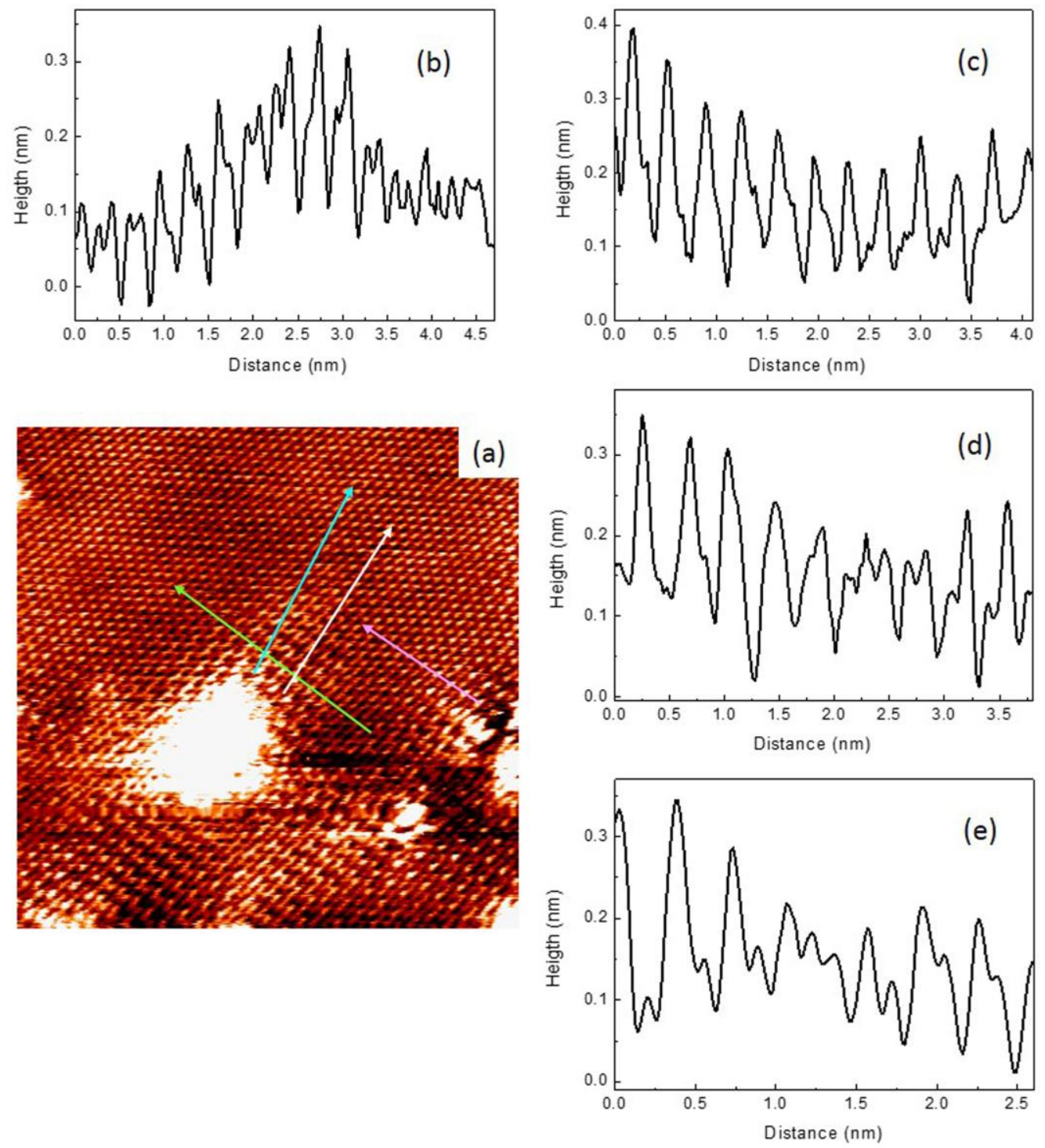

Figure S2 (a) STM image ( $\mathrm{V}_{\text {sample }}=+0.3 \mathrm{~V}$, Itunn $\left.=0.3 \mathrm{nA}\right)$ after one silicon monolayer deposited on the HOPG substrate at RT. We report the same STM image also in Figure 2(a) of the main text. Here we intend to underline the height differences between the silicene and the HOPG areas reporting several height profiles taken along (b) the green, (c) the cyan, (d) the white and (e) the magenta lines. Note that the height difference between the two regions is always around $0.15-0.20 \mathrm{~nm}$. 
(a) $G$ band

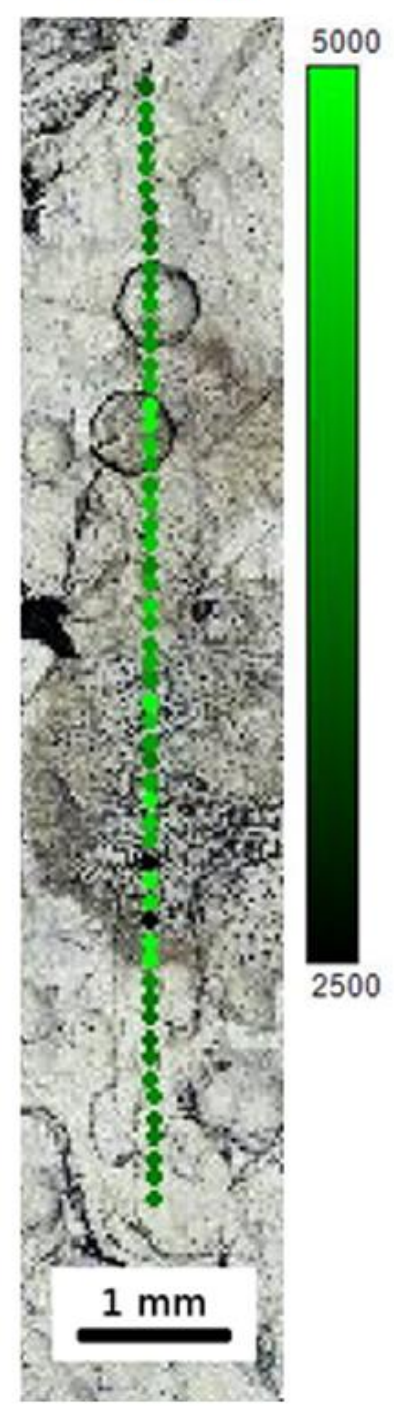

(b)

\section{$D$ band}

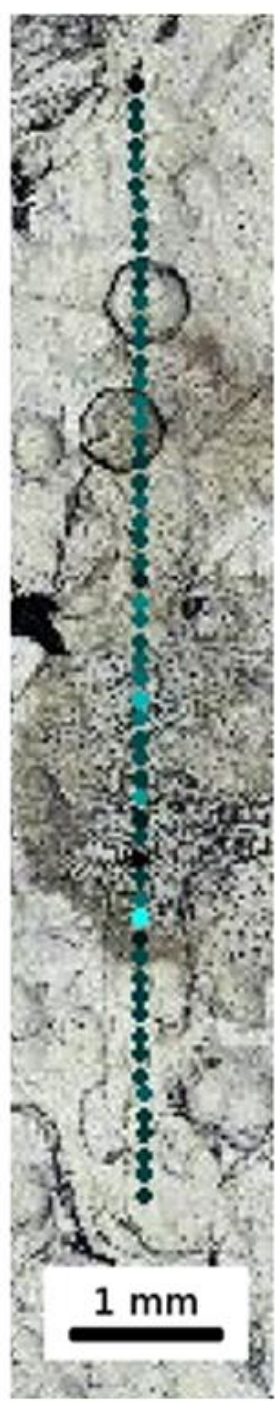

(c)

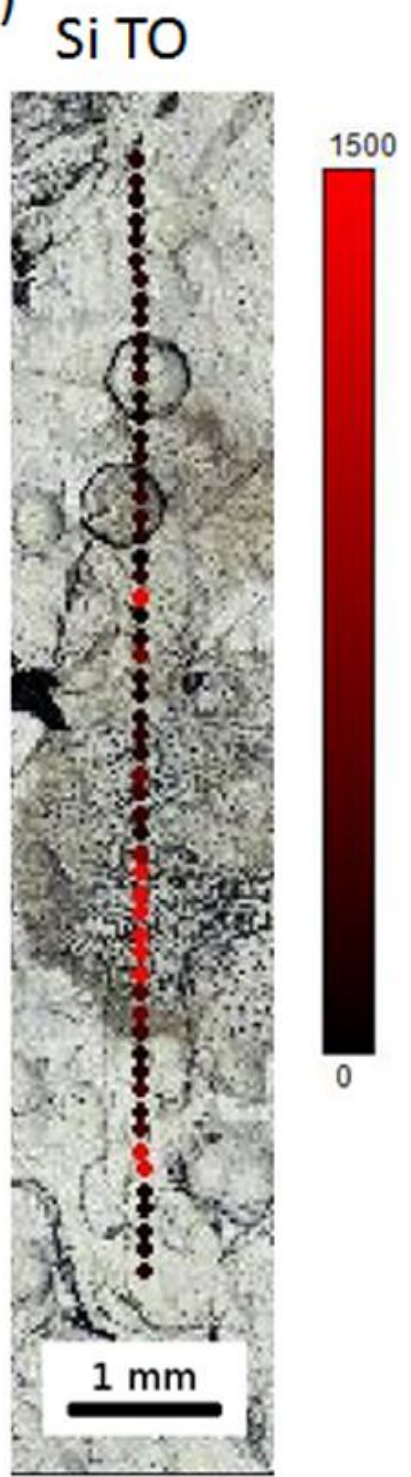

Figure S3. Low magnification (20X objective) Raman line scan across the whole HOPG surface highlighting the intensity distribution of the G Band (a, green dots), the D band (b, cyan dots), and Si TO (c, red dots). Color bars represent the intensity of the Raman features, the brightest colors correspond to the highest Raman intensity.

Low magnification Raman line-scan maps across the whole HOPG sample (Fig. S2). In such maps, the colored dots correspond to the Raman feature measured in that particular point. The intensity of the color of each dot is evidenced by the scale bar reported on the right side of each map. We identify the three main Raman modes as the graphite G (Fig. S2(a), green dots) and D bands (Fig. S2(b), cyan dots), as well as the bulk silicon TO mode (Fig. S2(c), red dots) peaked at $1580 \mathrm{~cm}^{-1}, 1340 \mathrm{~cm}^{-1}$ and 520 $\mathrm{cm}^{-1}$, respectively. Typical Raman spectra of bare HOPG and silicon decorated HOPG surface, extrapolated from Fig. S2 are reported in Fig. S3. 


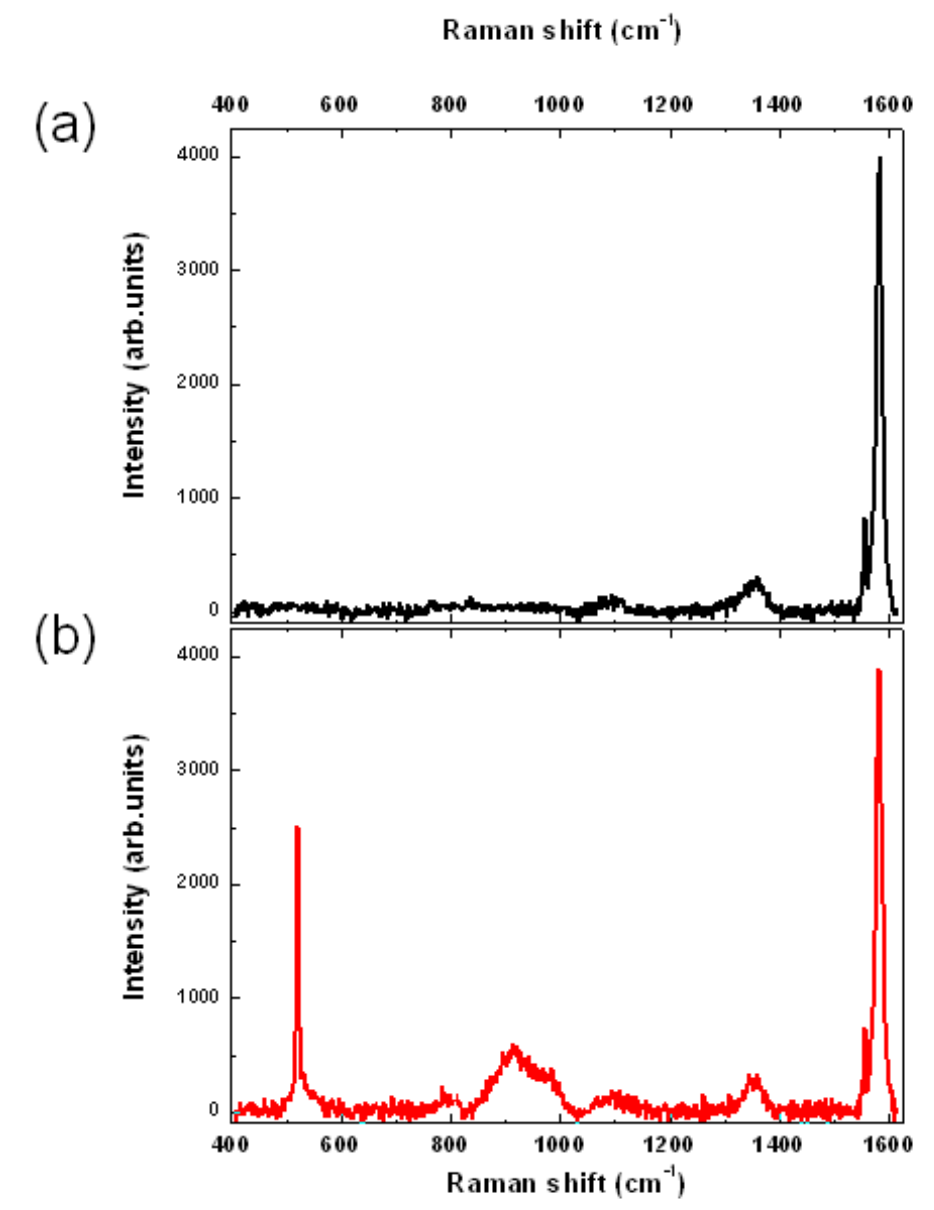

Figure S4 Low magnification Raman spectra extrapolated from Fig S1, (a) bare graphite, (b) silicon decorated graphite 


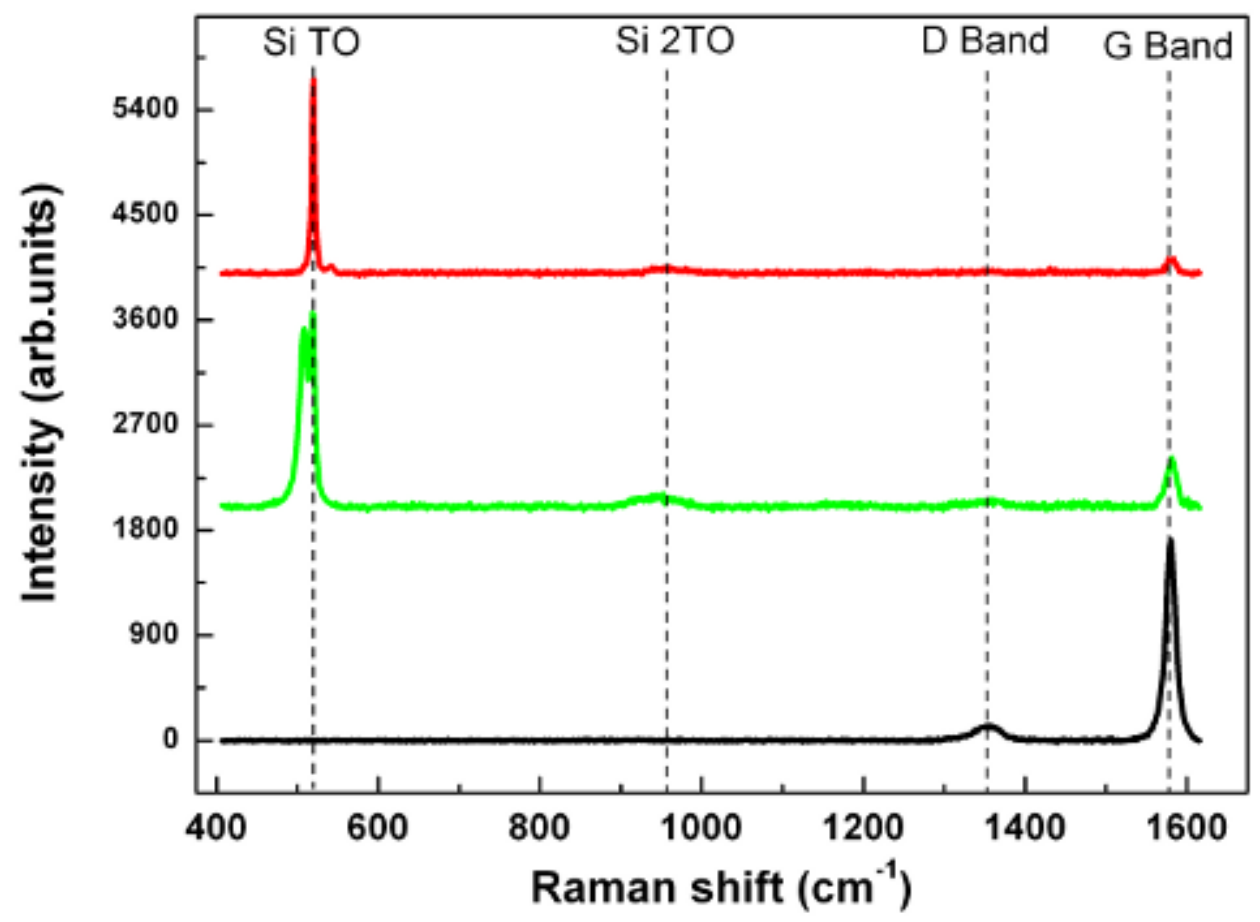

Figure S5 Raman spectra reported in Figure 3, but on the whole range of measured Raman shifts. 

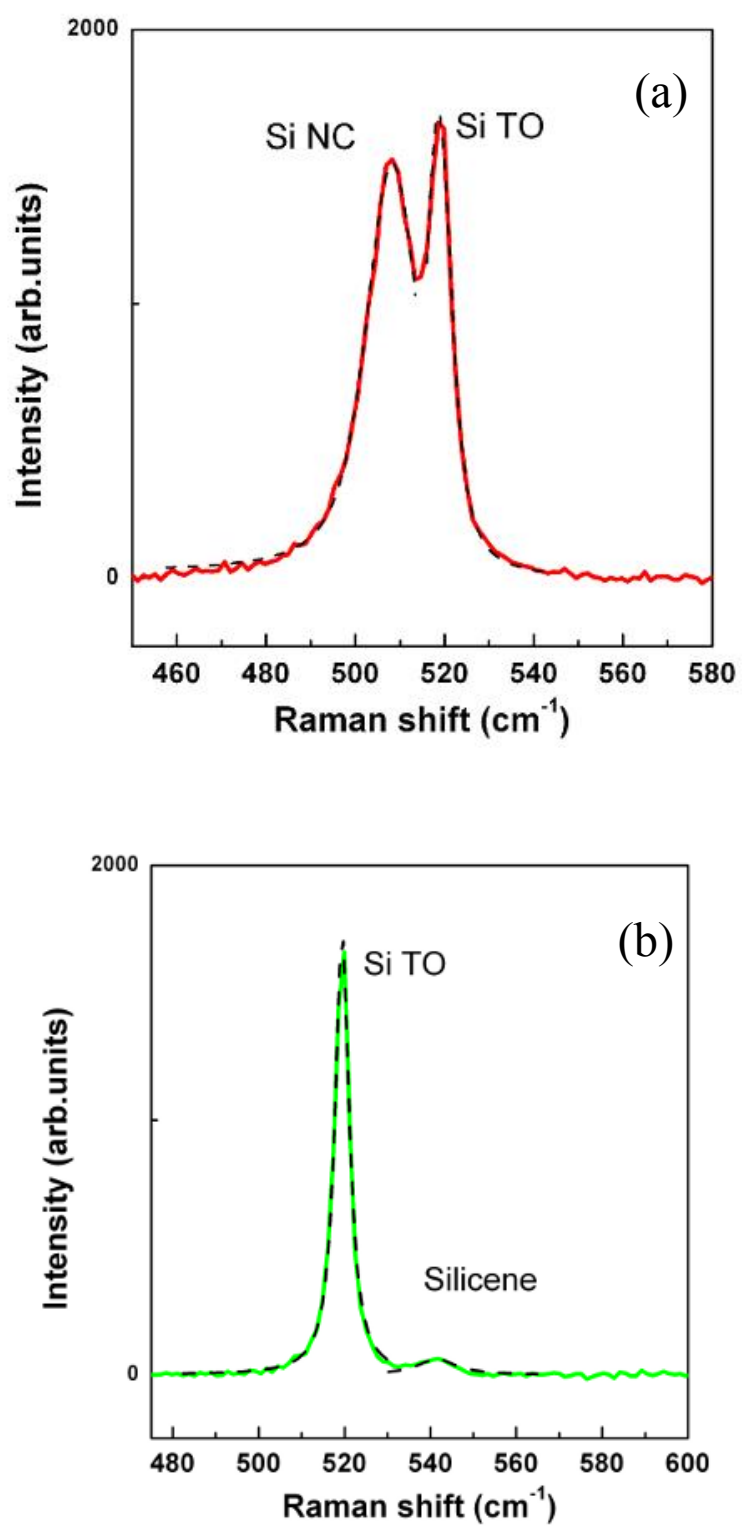

Figure S6 Voigt deconvolution of the Raman spectra reported in Figure 3b of the main text. In S5(a) Si nanocluster and Si large cluster modes are visible, while in S5(b) silicene mode located at $542.5 \mathrm{~cm}^{-1}$ is reported together with the TO Si large cluster. Peak positions and full half maximum widths are reported in Table S1.

\begin{tabular}{|l|c|c|c|}
\hline & Position $\left(\mathrm{cm}^{-1}\right)$ & Width $\left(\mathrm{cm}^{-1}\right)$ & Gaus (\%) \\
\hline Si NC & 510.0 & 13.8 & 29 \\
\hline Silicene & 542.5 & 7.2 & 100 \\
\hline Si TO & 519.8 & 5 & 22 \\
\hline
\end{tabular}

Table S1 Voigt deconvolution parameters of the Raman spectra shown in Figure 4: peak position, full width half maximum and Gaussian percentage. The fitting of the several peaks are reported in Figure S5 of the ESM. 


\section{Basis Set for $A b$ Initio Calculations}

The software that was used to calculate the Raman spectra uses Gaussian-type functions (GTF) as a basis set. All three atom types, $\mathrm{C}, \mathrm{H}$, and $\mathrm{Si}$, were described by an all-electron basis. For carbon ${ }^{1,2}$ we used a contraction of $6 s-211 s p-1 d^{*}$ GTFs (i.e. a contraction of $6,2,1,1$, and 1 GTFs for the $1 s, 2 s p$, 3sp, 4sp, and $d$ shells, respectively); for hydrogen ${ }^{3}$ a contraction of $511 s-1 p^{*}$ GTFs, and for $\mathrm{Si}^{4,5}$ a contraction of $8 s-6 s p-$ $211 s p-1 d^{*}$ GTFs. The outer exponents of $C$ were optimized for graphite using an energy criterion and are reported in Table S1. For Si, the outer exponents were optimized for silicene. For hydrogen, the outer exponents were optimized for $\mathrm{Si}_{2} \mathrm{H}_{6}$ using the optimized $\mathrm{Si}$ basis. The basis set optimizations were carried out using the LoptCG script ${ }^{6}$, which performs numerical gradient optimizations based on the conjugate gradient method. All optimized outer exponents are given in Table S2-S4.

Table S2: Exponents and coefficients of the contracted Gaussian basis set adopted in the present study for C. Only outer $s$ and $p$ GTF are given (see Ref. ${ }^{1,2}$ for a complete set of data).

\begin{tabular}{|c|c|c|c|}
\hline Shell & Exponent & Coefficient & \\
\hline & & $s(d)$ & $p$ \\
\hline$s p$ & 11.705961 & -0.160393 & 0.296329 \\
\hline & 2.877171 & -0.645825 & 1.383579 \\
\hline & 0.792605 & 1.0 & 1.0 \\
\hline$d$ & 0.234684 & 1.0 & \\
\hline & 0.927994 & 1.0 & \\
\hline
\end{tabular}

Table S3: Exponents and coefficients of the contracted Gaussian basis set adopted in the present study for $\mathrm{H}$. Only outer $s$ and $p$ GTF are given (see Ref. ${ }^{3}$ for a complete set of data).

\begin{tabular}{|c|c|c|}
\hline Shell & Exponent & Coefficient \\
\hline$s$ & 0.230254 & 1.0 \\
\hline & 0.083915 & 1.0 \\
\hline$p$ & 1.030824 & 1.0 \\
\hline
\end{tabular}


Table S4: Exponents and coefficients of the contracted Gaussian basis set adopted in the present study for Si. Only outer $s$ and $p$ GTF are given (see Ref. ${ }^{4,5}$ for a complete set of data).

\begin{tabular}{|c|c|c|c|}
\hline Shell & Exponent & Coefficient & \\
\hline & & $s(d)$ & $p$ \\
\hline$s p$ & 4.177539 & -0.024456 & -0.075700 \\
\hline & 1.054864 & -0.212516 & -0.082266 \\
\hline & 0.344699 & 1.0 & 1.0 \\
\hline$d$ & 0.111260 & 1.0 & \\
\hline
\end{tabular}




\section{Determination of the binding energy}

The binding energy $E_{b}$ is defined as follows:

$$
E_{b}=E_{o p t} I F-\left(E_{o p t} G r+E_{o p t} s i\right)
$$

Here, $E_{o p t} I F$ is the energy of the optimized interface, $E_{o p t} \mathrm{Gr}$ is the energy of the optimized graphite subsystem, and $E_{o p t}{ }^{S i}$ is the energy of the optimized silicene subsystem. 


\section{Determination of Raman-active Frequencies Obtained From Ab Initio Calculations}

For free-standing silicene the Raman-active $E_{2 g}$ mode can easily be determined either by the selection rules imposed by the symmetry of the system or by explicitly calculating the polarizability tensor to obtain the Raman intensities. Creation of HOPG/silicene interfaces or silicene nano-islands leads to a decrease or complete loss of symmetry, thus simply applying the selection rules is no longer possible. Furthermore, addition of HOPG to the system leads to a change in the band structure and the band gap is closed. However, the determination of the polarizability tensor is a perturbational approach (as implemented in the used software), which can break down in the case of no band gap. Thus the two standard methods to determine Raman activity are no longer viable.

Our approach relies on the assumption that the Raman active modes are the same (or very close) in freestanding silicene and the interface or nano-island systems. This assumption seems reasonable since no strong bonds are formed across the interface which would perturb the silicene system significantly; in fact the system is only held together by weak van-der-Waals interactions. The eigenvectors associated with the Raman active $E_{2 g}$ modes (in the following called displacement vectors) of free-standing silicene are readily available from a frequency calculation. By using these displacement vectors as a reference, we can therefore select the Raman active modes of the interfaces / nano-islands by calculating the overlap (i.e. the scalar product) between the reference displacement vector and the displacement vectors of the interfaces / nano-islands. The reference vector is constructed such that each top and bottom Si atom (with respect to the buckling) gets assigned the three Cartesian components of the displacement vector of the top and bottom $\mathrm{Si}$ atom of free-standing silicene. The contribution of the hydrogen atoms is neglected for the nano-islands. To increase the overlap and demonstrate the effectiveness of this approach, we also apply this method to a core region of $16 \mathrm{Si}$ atoms (see Fig. S6) for the nano-islands, where the modes are less disturbed by edge effects.

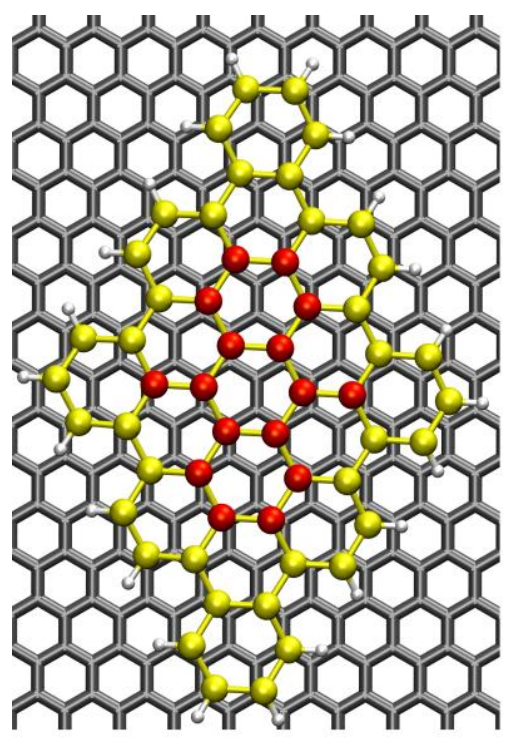

(a)

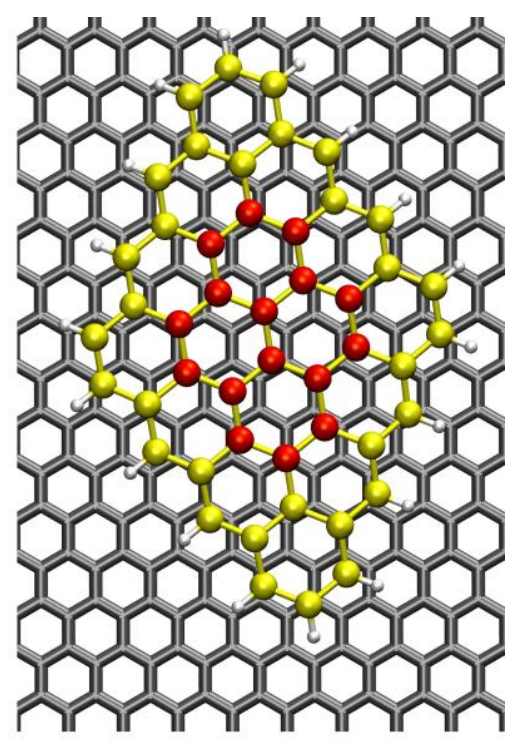

(b)

Figure S7 Core region (in red) for the overlap calculation of the (a) armchair edge and (b) zigzag edge nano-islands

Calculation of a spectrum for a system with $\mathrm{N}$ atoms leads to (3N-3) modes, however a multitude of those is either silent or barely active. In our case, we obtain about 30 modes in the region of $500-600 \mathrm{~cm}^{-1}$, many of them being in the critical region of $520-550 \mathrm{~cm}^{-1}$. In Tables S4-S9 we show the overlap obtained for all modes in the range of 500-600 $\mathrm{cm}^{-1}$ which clearly shows the effectiveness of this approach. The modes with maximum overlap are well separated from other modes. For the interfaces the maximum overlap is $>0.75$ in all cases, for a rotation angle of 10 degrees it is 1.00 . Most other modes show almost zero overlap, the second highest overlap (which does not stem from the two selected modes) is lower by 0.4. For the nano-islands the results are not as well separated, when the whole nano-island is considered. This is due to the inclusion of edge atoms which have a different chemical environment. However, the maximum overlap is still well above 0.5 and well separated from the other results (at least by 0.2 ). When 
only the core region is considered which has the same chemical environment as infinite silicene, the maximum overlap is pushed to $>0.75$, for a rotation angle of 10 degrees to even $>0.90$ for both armchair and zigzag edges. It also allows to clearly rule out the other modes, since the overlap is at least 0.5 lower. Figs. S7 and S8 show a comparison of the displacement vectors of the $E_{2 g}$ modes of free-standing silicene and the displacement vectors with maximum overlap for the nano-islands and also confirms our findings by visual inspection. 


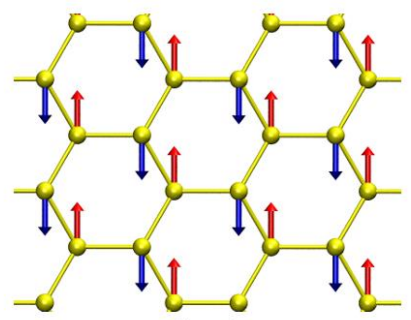

(a)

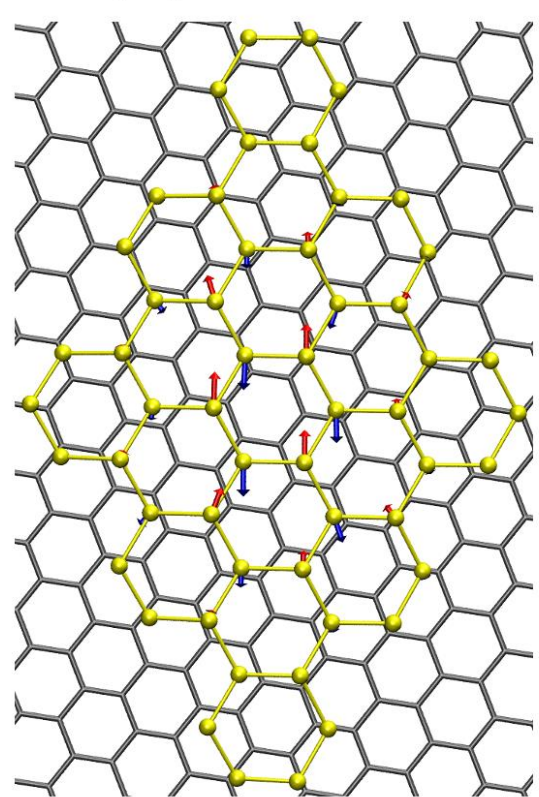

(d)

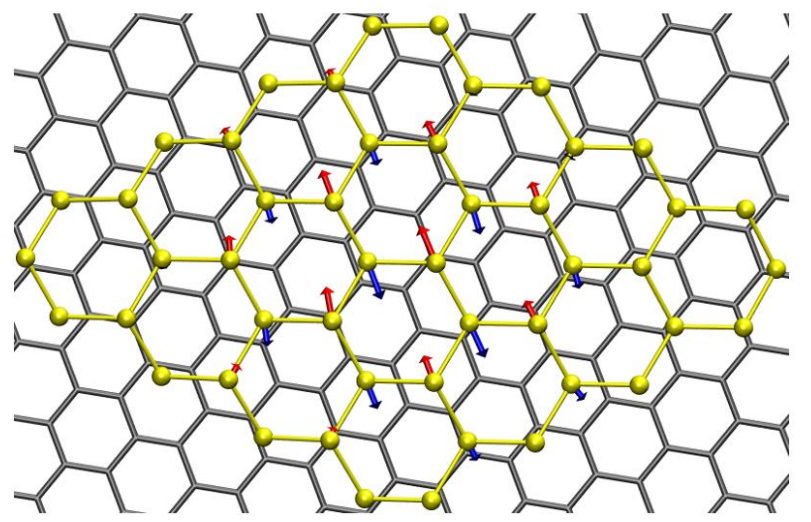

(f)

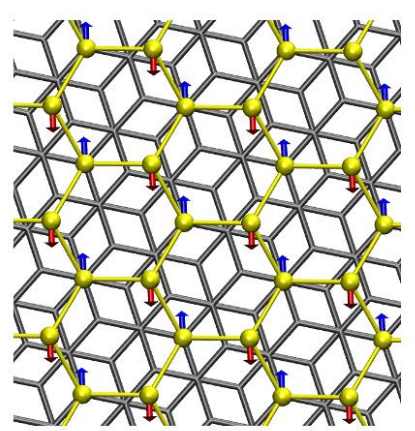

(b)

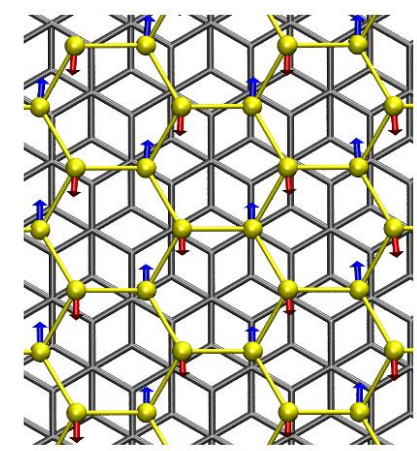

(c)

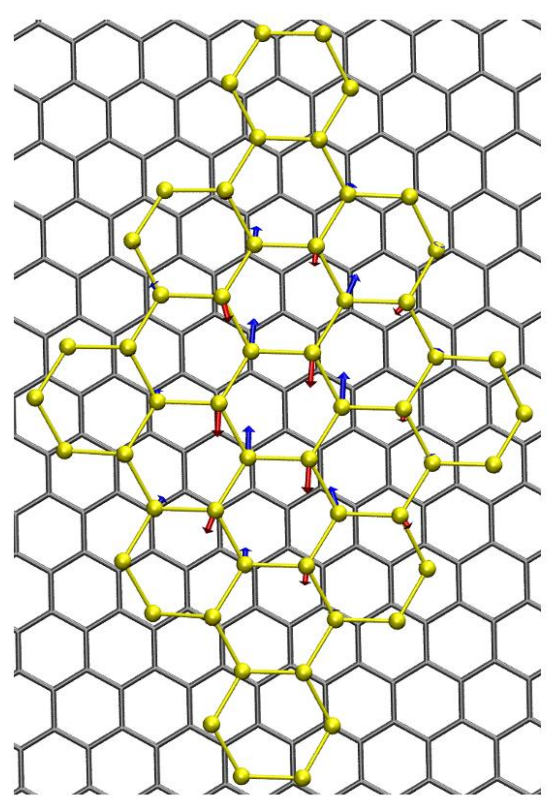

(e)

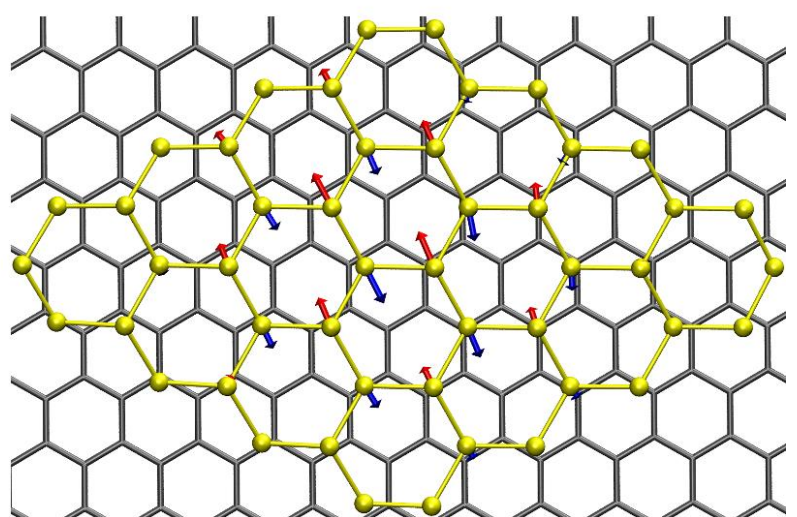

(g)

Figure S8 Displacement vectors for vibrations associated with mode 1 of free-standing silicene for the studied systems: (a) free-standing silicene, interfaces with (b) 10 and (c) 30 degrees rotation angle, armchair edge nanoislands with (d) 10 and (e) 30 degrees rotation angle, and zigzag edge nano-islands with (d) 10 and (e) 30 degrees rotation angle. 


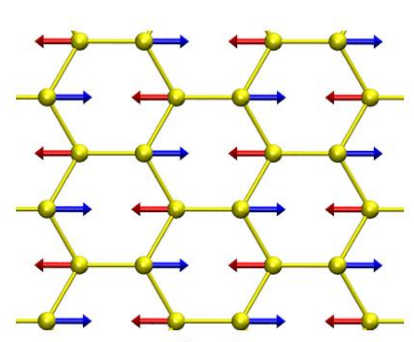

(a)

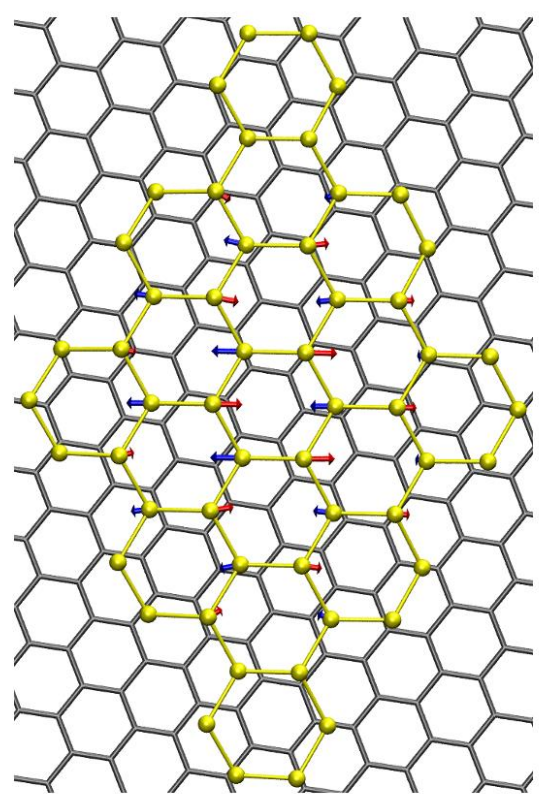

(d)

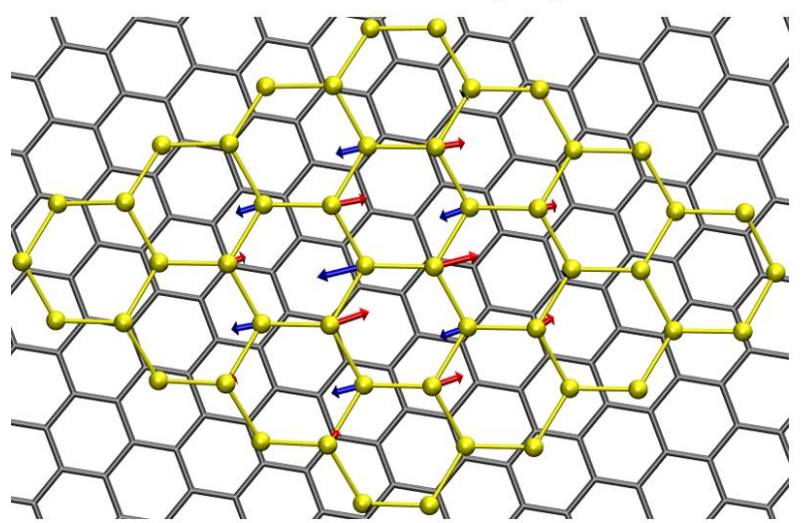

(f)

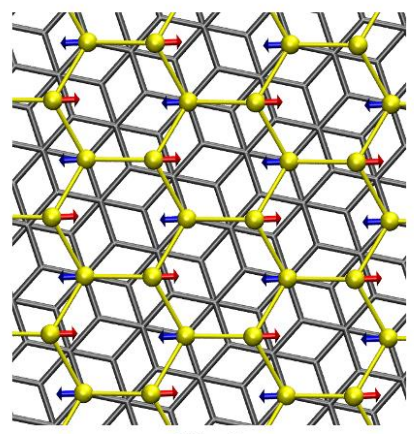

(b)

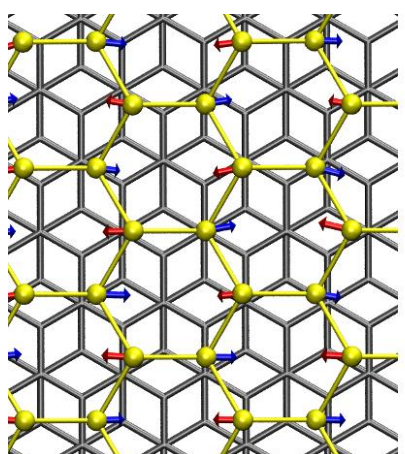

(c)

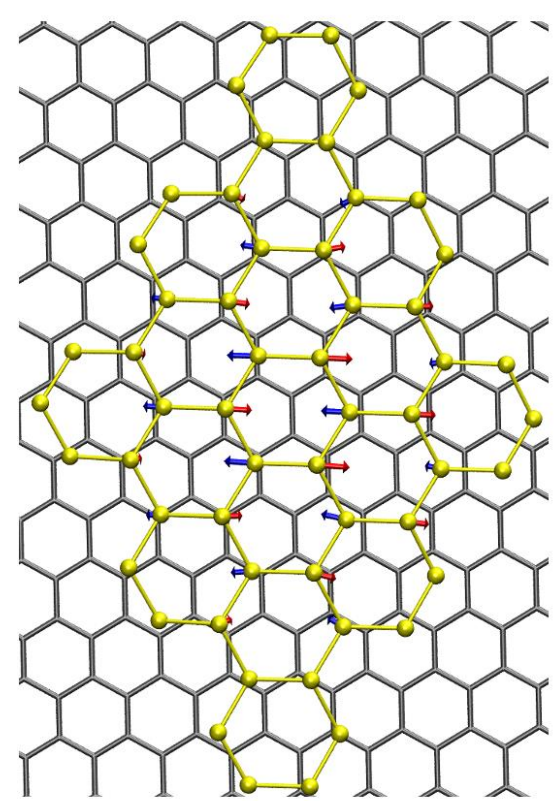

(e)

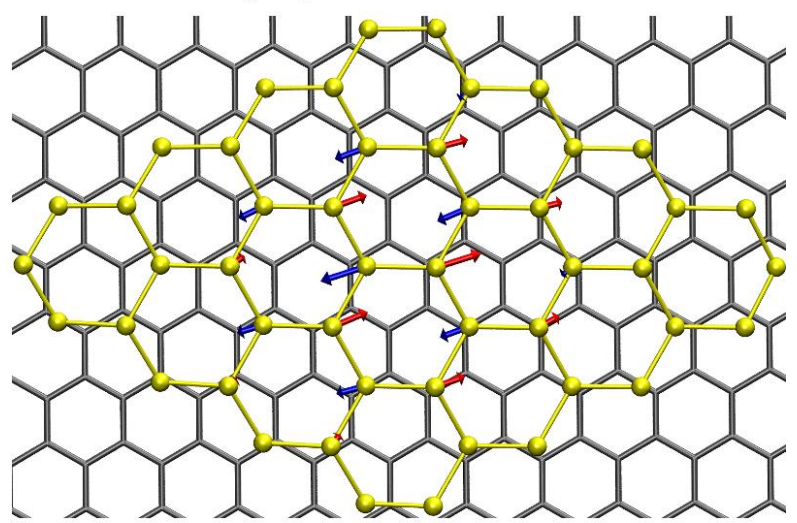

(g)

Figure S9 Displacement vectors for vibrations associated with mode 2 of free-standing silicene for the studied systems: (a) free-standing silicene, interfaces with (b) 10 and (c) 30 degrees rotation angle, armchair edge nanoislands with (d) 10 and (e) 30 degrees rotation angle, and zigzag edge nano-islands with (d) 10 and (e) 30 degrees rotation angle. 
Table S5 Absolute overlap for the HOPG/silicene interface at a rotation angle of 10 degrees. Mode 1 and 2 denote the degenerated $\mathrm{E}_{2 \mathrm{~g}}$ eigenvectors of free-standing silicene.

\begin{tabular}{|c|c|c|}
\hline Frequency $/ \mathrm{cm}^{-1}$ & Mode 1 & Mode 2 \\
\hline 536.84 & 0.017 & 0.007 \\
\hline 536.95 & 0.003 & 0.017 \\
\hline 582.89 & 0.006 & 1.000 \\
\hline 584.11 & 1.000 & 0.006 \\
\hline
\end{tabular}

Table S6 Absolute overlap for the HOPG/silicene interface at a rotation angle of 30 degrees. Mode 1 and 2 denote the degenerated $\mathrm{E}_{2 g}$ eigenvectors of free-standing silicene.

\begin{tabular}{|c|c|c|}
\hline Frequency $/ \mathrm{cm}^{-1}$ & Mode 1 & Mode 2 \\
\hline 500.09 & 0.000 & 0.002 \\
\hline 500.1 & 0.001 & 0.003 \\
\hline 505.98 & 0.001 & 0.000 \\
\hline 506 & 0.000 & 0.000 \\
\hline 506.05 & 0.000 & 0.001 \\
\hline 506.06 & 0.000 & 0.000 \\
\hline 506.08 & 0.001 & 0.001 \\
\hline 506.09 & 0.000 & 0.001 \\
\hline 532.85 & 0.002 & 0.003 \\
\hline 532.87 & 0.002 & 0.004 \\
\hline 532.92 & 0.007 & 0.005 \\
\hline 532.94 & 0.002 & 0.001 \\
\hline 533 & 0.002 & 0.001 \\
\hline 533.05 & 0.000 & 0.000 \\
\hline 537.41 & 0.031 & 0.017 \\
\hline 537.45 & 0.008 & 0.002 \\
\hline 537.86 & 0.006 & 0.003 \\
\hline 537.92 & 0.005 & 0.003 \\
\hline 537.93 & 0.010 & 0.002 \\
\hline 537.97 & 0.027 & 0.016 \\
\hline 539.43 & 0.002 & 0.000 \\
\hline
\end{tabular}




\begin{tabular}{|c|c|c|}
\hline 539.45 & 0.021 & 0.011 \\
\hline 539.52 & 0.066 & 0.039 \\
\hline 539.53 & 0.007 & 0.006 \\
\hline 539.58 & 0.010 & 0.006 \\
\hline 539.64 & 0.106 & 0.062 \\
\hline 539.79 & 0.852 & 0.494 \\
\hline 543.65 & 0.011 & 0.004 \\
\hline 543.67 & 0.004 & 0.013 \\
\hline 543.7 & 0.006 & 0.014 \\
\hline 543.72 & 0.010 & 0.006 \\
\hline 543.74 & 0.005 & 0.008 \\
\hline 543.78 & 0.006 & 0.008 \\
\hline 546.32 & 0.500 & 0.862 \\
\hline 555.1 & 0.039 & 0.025 \\
\hline 555.13 & 0.018 & 0.025 \\
\hline 555.5 & 0.007 & 0.049 \\
\hline 555.52 & 0.018 & 0.023 \\
\hline 555.53 & 0.046 & 0.029 \\
\hline 555.57 & 0.005 & 0.024 \\
\hline
\end{tabular}


Table S7 Absolute overlap of the HOPG/silicene nano-island with armchair edge at a rotation angle of 10 degrees. Overlap given for all Si atoms as well as for the core region. Mode 1 and 2 denote the degenerated E2g eigenvectors of free-standing silicene

\begin{tabular}{|c|c|c|c|c|}
\hline & \multicolumn{2}{|c|}{ Full Nano-island } & \multicolumn{2}{|c|}{ Core Region } \\
\hline Frequency $/ \mathrm{cm}^{-1}$ & Mode 1 & Mode 2 & Mode 1 & Mode 2 \\
\hline 504.35 & 0.002 & 0.050 & 0.036 & 0.289 \\
\hline 507.39 & 0.039 & 0.116 & 0.028 & 0.261 \\
\hline 508.52 & 0.024 & 0.022 & 0.019 & 0.028 \\
\hline 508.78 & 0.052 & 0.033 & 0.007 & 0.003 \\
\hline 510.54 & 0.062 & 0.010 & 0.056 & 0.008 \\
\hline 510.87 & 0.194 & 0.048 & 0.220 & 0.063 \\
\hline 511.76 & 0.074 & 0.014 & 0.083 & 0.006 \\
\hline 513.08 & 0.062 & 0.147 & 0.050 & 0.242 \\
\hline 515.84 & 0.022 & 0.060 & 0.081 & 0.031 \\
\hline 516.83 & 0.095 & 0.022 & 0.088 & 0.022 \\
\hline 517.75 & 0.002 & 0.113 & 0.028 & 0.177 \\
\hline 519.74 & 0.068 & 0.048 & 0.018 & 0.033 \\
\hline 520.1 & 0.142 & 0.058 & 0.037 & 0.062 \\
\hline 520.4 & 0.162 & 0.019 & 0.011 & 0.005 \\
\hline 521.18 & 0.223 & 0.038 & 0.049 & 0.009 \\
\hline 521.4 & 0.037 & 0.001 & 0.128 & 0.004 \\
\hline 521.61 & 0.143 & 0.074 & 0.088 & 0.001 \\
\hline 522.42 & 0.155 & 0.005 & 0.159 & 0.031 \\
\hline 524.61 & 0.044 & 0.123 & 0.023 & 0.117 \\
\hline 525.45 & 0.029 & 0.174 & 0.042 & 0.413 \\
\hline 525.55 & 0.041 & 0.007 & 0.159 & 0.183 \\
\hline 525.81 & 0.014 & 0.045 & 0.351 & 0.084 \\
\hline 526.19 & 0.016 & 0.150 & 0.127 & 0.170 \\
\hline 527.43 & 0.007 & 0.001 & 0.014 & 0.070 \\
\hline 531.07 & 0.011 & 0.020 & 0.001 & 0.007 \\
\hline 532.27 & 0.141 & 0.002 & 0.239 & 0.002 \\
\hline 532.55 & 0.012 & 0.021 & 0.018 & 0.086 \\
\hline
\end{tabular}




\begin{tabular}{|l|l|l|l|l|}
\hline 539.79 & 0.005 & 0.019 & 0.040 & 0.022 \\
\hline 542.38 & 0.654 & 0.067 & 0.939 & 0.101 \\
\hline 546.74 & 0.018 & 0.062 & 0.024 & 0.107 \\
\hline 550.83 & 0.078 & 0.720 & 0.104 & 0.972 \\
\hline
\end{tabular}


Table S8 Absolute overlap for the HOPG/silicene nano-island with armchair edge at a rotation angle of 30 degrees. Overlap given for all Si atoms as well as for the core region. Mode 1 and 2 denote the degenerated $\mathrm{E}_{2 \mathrm{~g}}$ eigenvectors of free-standing silicene.

\begin{tabular}{|c|c|c|c|c|}
\hline & Full Nano-island & Core Region & & \\
\hline Frequency $/ \mathrm{cm}^{-1}$ & Mode 1 & Mode 2 & Mode 1 & Mode 2 \\
\hline 503.81 & 0.014 & 0.010 & 0.248 & 0.173 \\
\hline 507.01 & 0.111 & 0.098 & 0.193 & 0.147 \\
\hline 508.01 & 0.018 & 0.012 & 0.014 & 0.005 \\
\hline 508.83 & 0.008 & 0.010 & 0.011 & 0.005 \\
\hline 510.14 & 0.018 & 0.012 & 0.039 & 0.033 \\
\hline 510.81 & 0.178 & 0.206 & 0.161 & 0.193 \\
\hline 511.68 & 0.007 & 0.041 & 0.000 & 0.028 \\
\hline 512.95 & 0.210 & 0.138 & 0.200 & 0.184 \\
\hline 515.37 & 0.004 & 0.006 & 0.025 & 0.047 \\
\hline 516.54 & 0.052 & 0.105 & 0.067 & 0.033 \\
\hline 517.67 & 0.051 & 0.050 & 0.126 & 0.132 \\
\hline 519.29 & 0.001 & 0.010 & 0.051 & 0.049 \\
\hline 519.67 & 0.006 & 0.033 & 0.014 & 0.026 \\
\hline 520.46 & 0.195 & 0.288 & 0.033 & 0.021 \\
\hline 520.74 & 0.107 & 0.188 & 0.004 & 0.034 \\
\hline 521.39 & 0.136 & 0.083 & 0.018 & 0.066 \\
\hline 521.81 & 0.036 & 0.066 & 0.041 & 0.053 \\
\hline 522.52 & 0.005 & 0.035 & 0.112 & 0.184 \\
\hline 524.84 & 0.087 & 0.019 & 0.203 & 0.098 \\
\hline 525.08 & 0.172 & 0.093 & 0.334 & 0.108 \\
\hline 525.65 & 0.022 & 0.014 & 0.026 & 0.013 \\
\hline 526.08 & 0.031 & 0.049 & 0.153 & 0.416 \\
\hline 526.19 & 0.007 & 0.046 & 0.005 & 0.372 \\
\hline 527.31 & 0.015 & 0.008 & 0.037 & 0.076 \\
\hline 530.78 & 0.003 & 0.005 & 0.003 & 0.013 \\
\hline 531.93 & 0.093 & 0.121 & 0.102 & 0.222 \\
\hline
\end{tabular}




\begin{tabular}{|l|l|l|l|l|}
\hline 532.47 & 0.027 & 0.038 & 0.076 & 0.016 \\
\hline 539.51 & 0.012 & 0.022 & 0.026 & 0.033 \\
\hline 542.14 & 0.405 & 0.525 & 0.576 & 0.747 \\
\hline 546.49 & 0.053 & 0.027 & 0.103 & 0.055 \\
\hline 550.48 & 0.603 & 0.461 & 0.779 & 0.592 \\
\hline
\end{tabular}


Table S9 Absolute overlap for the HOPG/silicene nano-island with zigzag edge at a rotation angle of 10 degrees. Overlap given for all Si atoms as well as for the core region. Mode 1 and 2 denote the degenerated $\mathrm{E}_{2 \mathrm{~g}}$ eigenvectors of free-standing silicene.

\begin{tabular}{|c|c|c|c|c|}
\hline & Full Nano-island & Core Region & & \\
\hline Frequency $/ \mathrm{cm}^{-1}$ & Mode 1 & Mode 2 & Mode 1 & Mode 2 \\
\hline 502.13 & 0.020 & 0.014 & 0.014 & 0.004 \\
\hline 503.46 & 0.164 & 0.150 & 0.062 & 0.063 \\
\hline 504.28 & 0.142 & 0.165 & 0.121 & 0.161 \\
\hline 505.09 & 0.093 & 0.140 & 0.103 & 0.148 \\
\hline 510.74 & 0.078 & 0.101 & 0.021 & 0.031 \\
\hline 511.06 & 0.162 & 0.076 & 0.003 & 0.012 \\
\hline 511.91 & 0.029 & 0.030 & 0.001 & 0.009 \\
\hline 512.33 & 0.061 & 0.023 & 0.023 & 0.041 \\
\hline 515.00 & 0.271 & 0.197 & 0.198 & 0.019 \\
\hline 515.07 & 0.126 & 0.231 & 0.061 & 0.067 \\
\hline 515.65 & 0.154 & 0.177 & 0.182 & 0.128 \\
\hline 518.63 & 0.005 & 0.020 & 0.082 & 0.119 \\
\hline 519.01 & 0.032 & 0.023 & 0.009 & 0.005 \\
\hline 520.28 & 0.004 & 0.012 & 0.103 & 0.101 \\
\hline 520.82 & 0.001 & 0.032 & 0.176 & 0.235 \\
\hline 523.03 & 0.184 & 0.116 & 0.082 & 0.094 \\
\hline 525.39 & 0.060 & 0.022 & 0.012 & 0.029 \\
\hline 525.74 & 0.039 & 0.021 & 0.035 & 0.043 \\
\hline 526.99 & 0.070 & 0.020 & 0.013 & 0.008 \\
\hline 528.35 & 0.006 & 0.037 & 0.002 & 0.020 \\
\hline
\end{tabular}




\begin{tabular}{|l|l|l|l|l|}
\hline 529.24 & 0.138 & 0.089 & 0.154 & 0.106 \\
\hline 530.62 & 0.130 & 0.011 & 0.168 & 0.087 \\
\hline 530.98 & 0.094 & 0.048 & 0.036 & 0.027 \\
\hline 532.46 & 0.054 & 0.123 & 0.050 & 0.087 \\
\hline 534.21 & 0.212 & 0.175 & 0.125 & 0.008 \\
\hline 535.89 & 0.016 & 0.007 & 0.017 & 0.056 \\
\hline 544.93 & 0.095 & 0.027 & 0.198 & 0.940 \\
\hline 546.20 & 0.242 & 0.645 & 0.260 & 0.289 \\
\hline 546.49 & 0.570 & 0.139 & 0.900 & \\
\hline
\end{tabular}


Table S10 Absolute overlap for the HOPG/silicene nano-island with zigzag edge at a rotation angle of 30 degrees. Overlap given for all $\mathrm{Si}$ atoms as well as for the core region. Mode 1 and 2 denote the degenerated $\mathrm{E}_{2 \mathrm{~g}}$ eigenvectors of free-standing silicene.

\begin{tabular}{|c|c|c|c|c|}
\hline & Full Nano-island & Core Region & & \\
\hline Frequency $/ \mathrm{cm}^{-1}$ & Mode 1 & Mode 2 & Mode 1 & Mode 2 \\
\hline 501.27 & 0.103 & 0.032 & 0.028 & 0.002 \\
\hline 503.78 & 0.178 & 0.160 & 0.039 & 0.077 \\
\hline 504.03 & 0.153 & 0.145 & 0.098 & 0.126 \\
\hline 504.25 & 0.117 & 0.225 & 0.110 & 0.181 \\
\hline 510.13 & 0.129 & 0.009 & 0.003 & 0.037 \\
\hline 510.62 & 0.142 & 0.114 & 0.005 & 0.003 \\
\hline 511.1 & 0.002 & 0.030 & 0.022 & 0.004 \\
\hline 511.96 & 0.036 & 0.028 & 0.043 & 0.033 \\
\hline 514.17 & 0.132 & 0.260 & 0.094 & 0.091 \\
\hline 514.29 & 0.294 & 0.197 & 0.186 & 0.018 \\
\hline 515.02 & 0.175 & 0.132 & 0.170 & 0.109 \\
\hline 517.92 & 0.026 & 0.013 & 0.076 & 0.132 \\
\hline 518.45 & 0.037 & 0.030 & 0.027 & 0.000 \\
\hline 519.73 & 0.029 & 0.004 & 0.029 & 0.084 \\
\hline 520.25 & 0.000 & 0.030 & 0.157 & 0.255 \\
\hline 522.51 & 0.208 & 0.105 & 0.095 & 0.051 \\
\hline 524.98 & 0.036 & 0.003 & 0.019 & 0.015 \\
\hline 525.1 & 0.011 & 0.016 & 0.002 & 0.016 \\
\hline 526.15 & 0.019 & 0.021 & 0.014 & 0.014 \\
\hline 527.94 & 0.019 & 0.011 & 0.012 & 0.004 \\
\hline 528.98 & 0.253 & 0.131 & 0.189 & 0.134 \\
\hline 529.4 & 0.094 & 0.102 & 0.120 & 0.069 \\
\hline 530.3 & 0.056 & 0.067 & 0.049 & 0.037 \\
\hline 531.69 & 0.100 & 0.017 & 0.098 & 0.023 \\
\hline 533.88 & 0.218 & 0.120 & 0.119 & 0.090 \\
\hline 535.1 & 0.003 & 0.002 & 0.003 & 0.011 \\
\hline 544.44 & 0.003 & 0.096 & 0.019 & 0.166 \\
\hline
\end{tabular}




\begin{tabular}{|c|l|l|l|l|}
\hline 545.5 & 0.128 & 0.676 & 0.124 & 0.963 \\
\hline 545.89 & 0.584 & 0.009 & 0.945 & 0.147 \\
\hline
\end{tabular}

\section{References}

[1] Dovesi, R.; Causà , M.; Orlando, R.; Roetti, C.; Saunders, V. R. Ab initio Approach to Molecular Crystals: A Periodic Hartree-Fock Study of Crystalline Urea. J. Chem. Phys. 1990, 92, 7402.

[2] Catti, M.; Pavese, A.; Dovesi, R.; Saunders, V. R. Static Lattice and Electron Properties of $\mathrm{MgCO}_{3}$ (Magnesite) Calculated by Ab Initio Periodic Hartree-Fock Methods. Phys. Rev. B: Condens. Matter Mater. Phys. 1993, 47, 9189-9198.

[3] Pascale, F.; Zicovich-Wilson, C. M.; Orlando, R. ; Roetti, C. ; Ugliengo, P. ; Dovesi, R. Vibration frequencies of $\mathrm{Mg}_{3} \mathrm{Al}_{2} \mathrm{Si}_{3} \mathrm{O}_{12}$ pyrope. An ab initio study with the CRYSTAL code, J. Phys. Chem. B 2005, 109, 6146-6152.

[4] Noel, Y.; Catti, M.; D'Arco, P. ; Dovesi, R. The vibrational frequencies of forsterite $\mathrm{Mg}_{2} \mathrm{SiO}_{4}$ : an all-electron ab initio study with the CRYSTAL code, Phys. Chem. Minerals 2006, 33, 383-393.

[5] Lichanot, A.; Orlando, R.; Mallia, G.; Mrawa, M.; Dovesi, R. VOH center in magnesium oxide: an ab initio supercell study, Chem. Phys. Lett. 2000, 318, 240-246.

[6] Zicovich-Wilson, C. LoptCG (Shell Procedure for Numerical Gradient Optimization); Instituto de Technologia Quimica Valencia: Valencia, 2006. 